TRANSACTIONS OF THE

AMERICAN MATHEMATICAL SOCIETY

Volume 349, Number 12, December 1997, Pages 4897-4930

S 0002-9947(97)01723-6

\title{
DEHN SURGERY ON KNOTS IN SOLID TORI CREATING ESSENTIAL ANNULI
}

\author{
CHUICHIRO HAYASHI AND KIMIHIKO MOTEGI
}

\begin{abstract}
Let $M$ be a 3-manifold obtained by performing a Dehn surgery on a knot in a solid torus. In the present paper we study when $M$ contains a separating essential annulus. It is shown that $M$ does not contain such an annulus in the majority of cases.

As a corollary, we prove that symmetric knots in the 3-sphere which are not periodic knots of period 2 satisfy the cabling conjecture. This is an improvement of a result of Luft and Zhang.

We have one more application to a problem on Dehn surgeries on knots producing a Seifert fibred manifold over the 2-sphere with exactly three exceptional fibres.
\end{abstract}

\section{INTRODUCTION}

Let $W=S^{1} \times D^{2}$ be a solid torus and $K$ a knot in $W$ which is not contained in a 3 -ball in $W$. Then we address the following question: when can we obtain a 3 -manifold containing an essential (i.e., incompressible and $\partial$-incompressible) annulus by Dehn surgery on $K$ ? More generally we consider the corresponding question for a connected orientable 3 -manifold $W$ which has a compressible toral boundary component $T$, and a knot $K$ in the interior of $W$. Let $N(K)$ be a tubular neighbourhood of $K$ in $W$. For the isotopy class (slope) $\gamma$ of an essential simple closed curve on $\partial N(K), W(K ; \gamma)$ denotes the 3 -manifold obtained from $W$ by $\gamma$-surgery on $K$, i.e., the result of attaching a solid torus $V$ to $W-\operatorname{int} N(K)$ by identifying $\partial V$ with $\partial N(K)$ so that $\gamma$ bounds a disc in $V$. We use $K^{*}$ to denote the core of $V$ in $W(K ; \gamma)$. Let $\mu$ be the meridian slope of $K$, and $\Delta=\Delta(\mu, \gamma)$ the minimal geometric intersection number of $\mu$ and $\gamma$. A $\operatorname{knot} K$ in $W$ (resp. $S^{3}$ ) is said to be cabled in $W$ (resp. $S^{3}$ ) if there is another knot (called a companion knot) $J$ in $W$ (resp. $S^{3}$ ) such that $K \subset \partial N(J)$ and $[K]=w[J] \in H_{1}(N(J) ; \mathbb{Z})$ with $|w| \geq 2$. A torus knot is also cabled in $S^{3}$ with a trivial companion knot.

Then our main results can be stated as follows.

Received by the editors June 28, 1995 and, in revised form, January 30, 1996

1991 Mathematics Subject Classification. Primary 57M25.

Key words and phrases. Dehn surgery, essential annulus, cabling conjecture, Seifert fibred manifold, Scharlemann cycle.

The first author was supported in part by Fellowships of the Japan Society for the Promotion of Science for Japanese Junior Scientists, and the second author was supported in part by Grantin-Aid for Encouragement of Young Scientists 07740077, The Ministry of Education, Science and Culture.

(C)1997 American Mathematical Society 
Theorem 1.1. Let $W$ be a connected orientable 3-manifold which has a compressible toral boundary compnent $T$, and $K$ a knot in $W$. Assume that $T$ is incompressible in $W-\operatorname{int} N(K)$. If $W(K ; \gamma)$ contains a separating essential annulus $A$ for $\Delta=\Delta(\mu, \gamma) \geq 4$, then either $(1) W(K ; \gamma)$ contains a separating essential annulus $A^{\prime}$ such that $A^{\prime} \cap K^{*}=\emptyset$ and $\partial A^{\prime}=\partial A$, or (2) the knots $K$ and $K^{*}$ are parallel to essential simple loops on $T$ in $W$ and $W(K ; \gamma)$ respectively, or $(3)$ the knot $K$ is cabled in $W$.

We give an example in Sect.12 which shows that Theorem 1.1 does not hold for $\Delta=2$.

If we require a special condition on the slope of a component of $\partial A(\subset \partial W(K ; \gamma))$, we have the following.

Theorem 1.2. Let $W, T$ and $K$ be as in Theorem 1.1, and let $D$ be a compressing disc of $T$ in $W$. If the manifold $W(K ; \gamma)$ contains a separating essential annulus $A$ such that each component of $\partial A$ intersects $\partial D$ transversely in one point, then $\Delta \leq 2$.

Let $W$ be a standardly embedded solid torus in $S^{3}$, and $K$ a knot in $W$. Then Dehn surgery on $K$ can be parametrized by rational numbers using a preferred meridian-longitude basis. The first assertion (1) of the next theorem follows from Theorem 1.2.

Theorem 1.3. Let $W$ be a standardly embedded solid torus in $S^{3}$ with a meridian disc $D$, and $K$ a knot in $W$ which is not contained in a 3-ball in $W$.

(1) If $W(K ; m / n)$ contains a separating essential annulus $A$ each of whose boundary components intersects $\partial D$ once, then $|n| \leq 2$.

(2) If $W(K ; m / n)$ contains a non-separating annulus $A$ each of whose boundary components is represented by $L+p M$, where $(M, L)$ denotes a preferred meridian-longitude pair of $W$ in $S^{3}$, then $m / n=p w^{2}(w=$ the algebraic intersection number of $K$ and $D)$. Moreover, $K$ can be trivialized by twisting $(-p)$-times along the meridian disc $D$.

Examples. (1) A family of knots in a solid torus $W$, such that integral surgeries on them produce 3-manifolds containing separating essential annuli with boundary as in Theorem 1.3, was given in [22, Lemma 9.1] (see Lemma 13.1 in this paper).

(2) If $K$ is the knot in $W$ depicted in Figure $1(1)$, then $W(K ; 0)$ contains a non-separating annulus with boundary as in Theorem 1.3.

Remark. Let $K$ be a $(1+2 p, 2)$-cable of a core of $W$ (see Figure $1(2)$ in the case $p=0)$; then both $W(K ; 4 p)$ and $W(K ; 4(p+1))$ contain non-separating annuli. Furthermore Theorem 1.3 (2) together with [19, Theorem 4.2] (see also [21]) shows that if two distinct surgeries on $K$ produce 3-manifolds containing non-separating annuli, then $K$ is a $(1+2 p, 2)$-cable of a core of $W$.

Let us give sample applications of our results.

The cabling conjecture ([6]) states that only Dehn surgeries on cabled knots in $S^{3}$ can produce reducible manifolds. It is known by [11] that only integral surgeries can produce reducible manifolds.

In [20], Luft and Zhang proved a weaker version of Theorem 1.3 (1) and proved the cabling conjecture for symmetric knots which are not periodic knots of period 2,3 or 5 . For the convenience of the readers, we briefly explain their proof. 


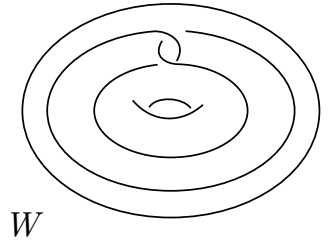

(1)

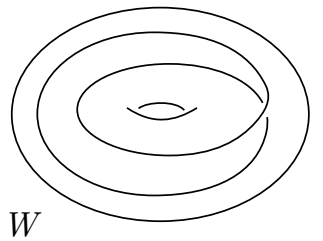

$(2)$

FiguRE 1

Let $\widetilde{K}$ be a non-cabled periodic knot with a periodic automorphism $f$ of $S^{3}$ of period $n$. Due to the positive answer to the Smith conjecture [24], $f$ is a rotation of $S^{3}$ and $F i x(f)$ (= the fixed point set of $f$ ) is a trivial knot disjoint from $\widetilde{K}$. Hence $\widetilde{K}$ is contained in the unknotted solid torus $\widetilde{W}=S^{3}-\operatorname{int} N($ Fix $(f))$, where $N($ Fix $(f))$ is an $f$-invariant tubular neighbourhood of Fix $(f)$ in $S^{3}$. Passing through the branched covering $p: S^{3} \rightarrow S^{3} /\{f\}\left(\cong S^{3}\right)$, we obtain the factor knot $K=p(\widetilde{K})$ $\left(\subset S^{3}\right)$. We note that $K$ is contained in the unknotted solid torus $W=p(\widetilde{W})$, and is not cabled because $\widetilde{K}$ is a non-cabled knot. (Since $\widetilde{K}$ and $K$ are knots in $S^{3}$, surgery slopes are parametrised by rational numbers using a preferred meridian-longitude pair.) Luft and Zhang ([20]) showed that if $\widetilde{K}$ produces a reducible manifold by $m$-surgery, then $W(K ; m / n)$ contains a separating essential annulus each of whose boundary components intersects $\partial D(D$ is a meridian disc of $W)$ once. Furthermore they proved that if $K$ is not cabled, then $\Delta(m / n, 1 / 0)=|n| \leq 6$ ([20, Lemma]).

Applying Theorem 1.3 (1) instead of [20, Lemma], we have:

Theorem 1.4. Symmetric knots in $S^{3}$ which are not periodic knots of period 2 satisfy the cabling conjecture.

Recently Gordon and Luecke [14] announced that they had settled the cabling conjecture for symmetric knots. Independently, by a different method, Hayashi and Shimokawa [16] also settled the cabling conjecture for symmetric knots. In [16] Hayashi and Shimokawa proved the impossibility of $|n|=2$ in Theorem 1.3 (1) using Lemma 3.1 and graph theoretical arguments. As a result they showed that periodic knots of period 2 satisfy the cabling conjecture.

We also apply Theorem 1.1 to the following question.

Question. When can we obtain a Seifert fibred manifold by Dehn surgery on a knot in $S^{3}$ ?

If $K$ is a satellite knot which is not cabled exactly once, then only integral surgeries can yield Seifert fibred manifolds ([1], [22]); moreover if there are two such surgeries, then they are successive integers and hence there are at most two such surgeries [23]. For hyperbolic knots Boyer and Zhang [1] proved that only integral surgeries can produce non-simple Seifert fibred manifolds.

Let $K$ be a torus knot or a cable of a torus knot. Then infinitely many surgeries on $K$ produce Seifert fibred manifolds over the 2-sphere $S^{2}$ with three exceptional fibres. In these examples, one of three exceptional fibres is the image of a trivial knot in $S^{3}$. 
Theorem 1.5. Let $K$ be a knot in $S^{3}$ and $C$ a trivial knot disjoint from $K$. Suppose that $m / n$-surgery on $K$ yields a Seifert fibred manifold over $S^{2}$ with three exceptional fibres such that the image of $C$ in the resulting manifold is one of three exceptional fibres. If $|n| \geq 4$, then $K$ is a torus knot or a cable of a torus knot.

This paper is organized as follows. In Sect.2 we give terminology and preliminary lemmas. In Sect. 3 we show the utility of two Scharlemann cycles for distinct intervals. Sect. 4 consists of lemmas about interior edges. We prove Theorem 1.2 (and hence Theorem $1.3(1)$ ) in Sect.5. A proof of Theorem 1.3(2) is given in Sect.6. To handle the situation described in Theorem 1.1, we need some lemmas concerning boundary edges, which are given in Sect.7. Sect.8 contains a proof of Theorem 1.1 in the special case where the essential annulus $A$ in $W(K ; \gamma)$ intersects $K^{*}$ exactly twice. In this special case we obtain a stronger conclusion (Proposition 8.1). In Sects.9-11, we consider the general case. We divide this into three cases (see Sect.9, 10 and 11 respectively) and finally show that the conclusion of Theorem 1.1 holds or the above special case occurs. Hence we establish Theorem 1.1. In Sect.12 we exhibit an example which shows that Theorem 1.1 does not hold for $\Delta=2$. In the final section, Sect.13, we prove Theorem 1.5 and present related examples.

\section{Preliminaries}

We take the compressing disc $D$ of $T$ in $W$ so that $q_{D}=|D \cap K|$ is minimal over all compressing discs of $T$ in $W$. Note that all the compressing discs have the same boundary slopes on $T$ because $T$ is a torus. We have an incompressible and $\partial$-incompressible planar surface $P_{D}=D \cap(W-\operatorname{int} N(K))$ in $W-\operatorname{int} N(K)$.

We are given a boundary slope $\partial A$ on the torus $T$. We take the annulus $A$ in $W(K ; \gamma)$ so that $q_{A}=\left|A \cap K^{*}\right|$ is minimal over all essential annuli in $W(K ; \gamma)$ with the given slope as above. Then the surface $P_{A}=A \cap(W-\operatorname{int} N(K))$ is incompressible and $\partial$-incompressible in $W-\operatorname{int} N(K)$.

Hence we can further take the compressing disc $D$ and the annulus $A$ with the same boundary slopes on $T$ as before so that $\partial P_{D}$ and $\partial P_{A}$ intersect in minimal points and so that their intersection consists of loops and arcs which are essential on both $P_{D}$ and $P_{A}$.

As in [12] we will form graphs on $A$ and $D$.

In the following we assume that $\{i, j\}=\{A, D\}$. Assigning arbitrary orientations to $P_{i}$ allows us to refer to + and - boundary components of $\partial P_{i} \cap \partial N(K)$, according to the direction of the induced orientation of a boundary component as it lies on $\partial N(K)$.

We orient the knots $K$ and $K^{*}$ arbitrarily. Number the components of $\partial P_{i} \cap$ $\partial N(K),\left\{1,2, \ldots, q_{i}\right\}$ in the order in which they appear on $\partial N(K)$. We may assign the number " 1 " to an arbitrary component of $\partial P_{i}$. Thus $K$ and $K^{*}$ are divided into $q_{D}$ and $q_{A}$ intervals $[1,2],[2,3], \ldots,\left[q_{i}, 1\right]$.

Since $T$ is incompressible in the exterior of $K$, we have $q_{D} \geq 1$. If $q_{A}=0$, then we have $A \cap K^{*}=\emptyset$, and we are done for Theorem 1.1. For Theorem 1.2 we need the following lemma.

Lemma 2.1. A separating annulus $B$ properly embedded in $W$-int $N(K)$ with $\partial B$ $\subset T$ is $\partial$-compressible if each component of $\partial B$ intersects $\partial D$ transversely in one point. 


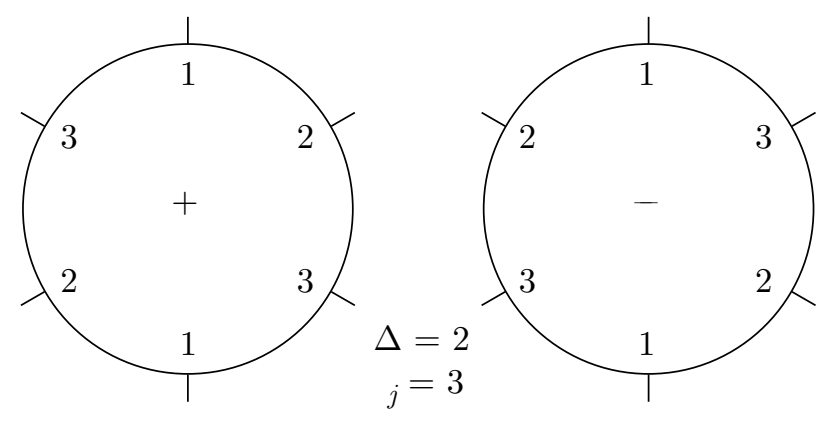

Figure 2

Proof. After an isotopy the intersection $B \cap P_{D}$ consists of one $\operatorname{arc} \alpha$ and loops. The $\operatorname{arc} \alpha$ forms an essential arc in the annulus $B$. Hence the loops of the intersection are inessential on the annulus $B$. Then since $P_{D}$ is incompressible, by a standard cut and paste argument we can choose the compressing disc $D$ so that $B \cap P_{D}$ consists of the $\operatorname{arc} \alpha$. The $\operatorname{arc} \alpha$ divides the disc $D$ into two discs, one of which does not intersect the knot $K$ because $B$ separates $W(K ; \gamma)$. This subdisc of $D$ forms a $\partial$-compressing disc of $B$ in $W-\operatorname{int} N(K)$.

Hence if $A \cap K^{*}=\emptyset$, then by the above lemma $A$ would be $\partial$-compressible in $W(K ; \gamma)$, a contradiction. We assume in the following that $q_{A} \geq 2$. Note that $q_{A}$ is even since $A$ is separating in $W(K ; \gamma)$.

We label the end points of arcs of $P_{A} \cap P_{D}$ in $P_{i}$ with the corresponding boundary components of $P_{j}$. Thus around each component of $\partial P_{i}$ we see the labels $\left\{1,2, \ldots, q_{j}\right\}$ appearing sequentially (either clockwise or anticlockwise according to the sign + or - of this component of $\left.\partial P_{i}\right) \Delta$ times. See Figure 2.

We regard the discs $i \cap V$ as forming the "fat vertices" of a graph $\Gamma_{i}$ in the surface $i$, the edges of $\Gamma_{i}$ corresponding to the arcs of $P_{A} \cap P_{D}$ in $P_{i}$ except for the arcs both of whose end points are in $\partial i$. We call the closure of a component of $\partial$ (fat vertex)-(end points of edges) a corner. If an edge $e$ connects a vertex to a vertex, then we say $e$ is an interior edge, otherwise a boundary edge. If an interior edge $e$ has both end points in the same fat vertex, then we say $e$ is a loop. The graph $\Gamma_{i}$ contains no trivial loops, i.e., 1-sided faces (no arc of $P_{A} \cap P_{D}$ is boundary parallel in $P_{i}$ ). Two edges $e$ and $e^{\prime}$ of $\Gamma_{i}$ are parallel if there is a disc $B$ in $P_{i}$ such that $\partial B=e \cup b \cup e^{\prime} \cup b^{\prime}$, where $b$ and $b^{\prime}$ are $\operatorname{arcs}$ in $\partial P_{i}$. Every fat vertex $v$ is assigned a sign + or - according to that of the loop $\partial v$. If an interior edge $e$ connects vertices of the same sign, then we say $e$ is a sign-preserving edge, otherwise a signreversing edge. A loop is a sign-preserving edge. Since $W-\operatorname{int} N(K)$ is orientable, we have the parity rule : an interior edge of the graph $\Gamma_{i}$ is a sign-preserving edge if and only if the corresponding edge of the other graph $\Gamma_{j}$ is a sign-reversing edge. We thus obtain two labeled graphs in $D$ and $A$, whose edges are in one to one correspondence. We call components of $i-\Gamma_{i}$ faces of $\Gamma_{i}$. A face $P$ is called a disc face if $P$ is an open disc. For every face $P$, let $\partial P$ denote its boundary, i.e., the subgraph which consists of vertices and edges intersecting $\bar{P}-P$.

Let $x$ be a label of $\Gamma_{i}$. An $x$-edge in $\Gamma_{i}$ is an interior edge with label $x$ at an end point. A subgraph $\sigma$ is an $x$-edge cycle if all its edges are sign-preserving $x$-edges 


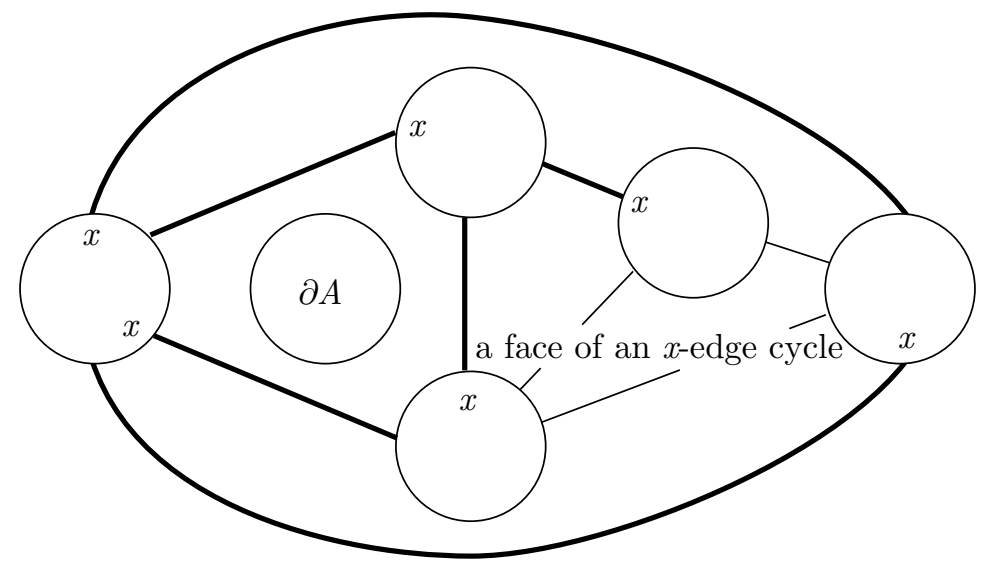

FIGURE 3

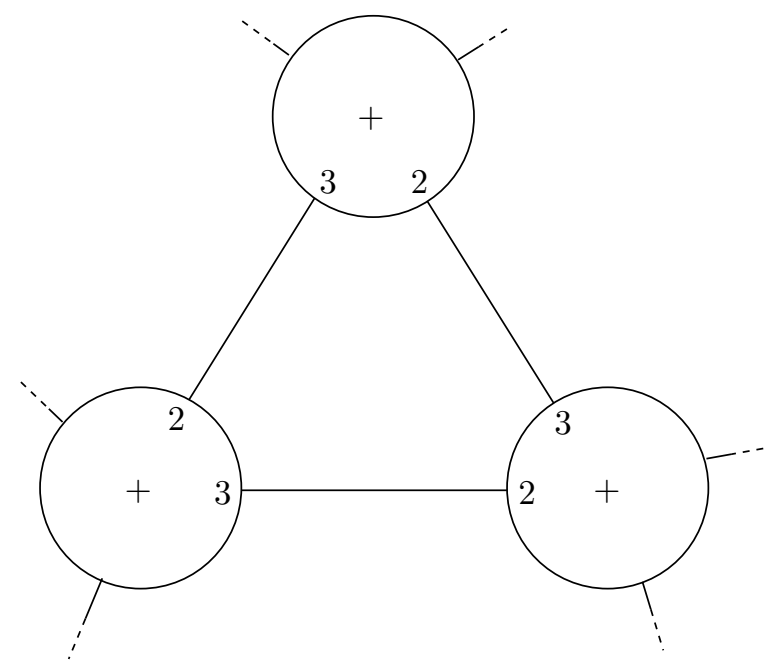

FiguRE 4

and if there is a disc face $P$ of the subgraph $\sigma$ such that $\sigma=\partial P . P$ is called the disc face of the $x$-edge cycle. See Figure 3.

If all the vertices in the disc face of an $x$-edge cycle $\sigma$ have the same sign as those of the vertices of the cycle, then we call $\sigma$ is a great $x$-edge cycle. A Scharlemann cycle is an $x$-edge cycle for some label $x$ which bounds a disc face of $\Gamma_{i}$. See Figure 4.

A Scharlemann cycle is a trivial loop if it consists of only one edge. Note that if $q_{D}=1$, then all the interior edges in $\Gamma_{D}$ are sign-preserving edges, and hence the parity rule implies that $\Gamma_{A}$ has no sign-preserving edges. In particular $\Gamma_{A}$ does not contain a Scharlemann cycle.

Lemma 2.2. Let $E$ be a disc face of a Scharlemann cycle $\sigma$ in $\Gamma_{i}$. Then the limit circle $c$ of the end of the open disc $E$ is embedded in the surface $i$, and two adjacent labels appear alternately on the circle $c$ as shown in Figure 4. 
Proof. The cycle $\sigma$ consists of at least two sign-preserving $x$-edges for some label $x$ because $\Gamma_{i}$ contains no trivial loop edges. Note that the limit circle $c$ is embedded in the surface $i$, since vertices are fat and $q_{j} \geq 2$ and $E$ is a face of $\Gamma_{i}$. Hence $c=\partial \bar{E}$. If an end point of a corner in $c$ has the label $x$, then the corner has the label $x-1$ or $x+1\left(\bmod q_{j}\right)$, say $x+1$ at the other end point. Then the edge incident to this end point with label $x+1$ has the label $x$ at the other end point because it is an $x$-edge. Similar arguments show that we see labels $x, x+1$ on the circle $c$ alternately.

We call a Scharlemann cycle with labels $x$ and $x+1$ a Scharlemann cycle for the interval $[x, x+1]$. For example, the Scharlemann cycle in Figure 4 is a Scharlemann cycle for the interval $[2,3]$.

Lemma 2.3. ([2, Lemma 2.5.2(a)]) The graph $\Gamma_{A}$ does not contain a Scharlemann cycle.

Proof. Suppose for a contradiction that $\Gamma_{A}$ has a Scharlemann cycle $\sigma$ for an interval $[x, x+1]$. Let $E$ be the disc face of $\sigma$. Note that $E \cap D=\emptyset$, since the intersection $P_{A} \cap P_{D}$ does not contain an inessential closed curve. Let $u, v$ be vertices of $\Gamma_{D}$ which are assigned the numbers $x, x+1$ respectively. The boundary components of $\partial P_{D}$ numbered $x, x+1$ are adjacent on the torus $\partial N(K)$. These boundary components cobound an annulus $Q$ containing all the corners in $c$. We obtain a new disc $D^{\prime}$ by performing surgery on the once punctured torus $(D-u-v) \cup Q$ along the disc $E$, because $c$ has non-zero algebraic intersection number with a core of $Q$. Since $\partial D^{\prime}=\partial D$, the disc $D^{\prime}$ is also a compressing disc of $T$; moreover $\left|D^{\prime} \cap K\right|=|D \cap K|-2$, which contradicts the minimality of $|D \cap K|$.

We call a surgery as in the above proof of Lemma 2.3 a surgery on $D$ along a Scharlemann cycle $\sigma$. We call the subgraph of $\Gamma_{D}$ consisting of the vertices $u$ and $v$ and the edges corresponding to those of $\sigma$ as in the above proof of Lemma 2.3 a Scharlemann co-cycle of $\sigma$.

\section{Two Scharlemann Cycles For Distinct intervals And $\partial$-INCOMPRESSIBILITY}

If $\Gamma_{A}$ contains a Scharlemann cycle, then the proof of Lemma 2.3 shows that we can take a compressing disc $D^{\prime}$ such that $\partial D^{\prime}=\partial D$ and $\left|D^{\prime} \cap K\right|<|D \cap K|$. In this section, we treat the case where $\Gamma_{D}$ contains a Scharlemann cycle. If $\Gamma_{D}$ contains a Scharlemann cycle, then can we find another essential annulus $A^{\prime}$ such that $\partial A^{\prime}=\partial A$ and $\left|A^{\prime} \cap K^{*}\right|<\left|A \cap K^{*}\right|$ ? It is not expected in general. Our purpose in this section is to prove the following lemma. In the proof we demonstrate that the existence of two Scharlemann cycles for distinct intervals allows us to find another essential annulus $A^{\prime}$ as above.

It should be noted that Gordon and Luecke make use of two Scharlemann cycles for distinct intervals in [13] to prove the "reducing conjecture".

Lemma 3.1. Suppose that $\Gamma_{D}$ contains two Scharlemann cycles $\sigma_{1}$ and $\sigma_{2}$ for distinct intervals $[x, x+1]$ and $[y, y+1]$ respectively. Then $q_{A}=2$.

To prove this, for a moment we consider the following situation. The notation used here is temporary.

Let $M$ be a connected, orientable 3-manifold with boundary. Let $T$ be a toral component of $\partial M$, and $A$ an annulus on $T$ such that its core is an essential loop 


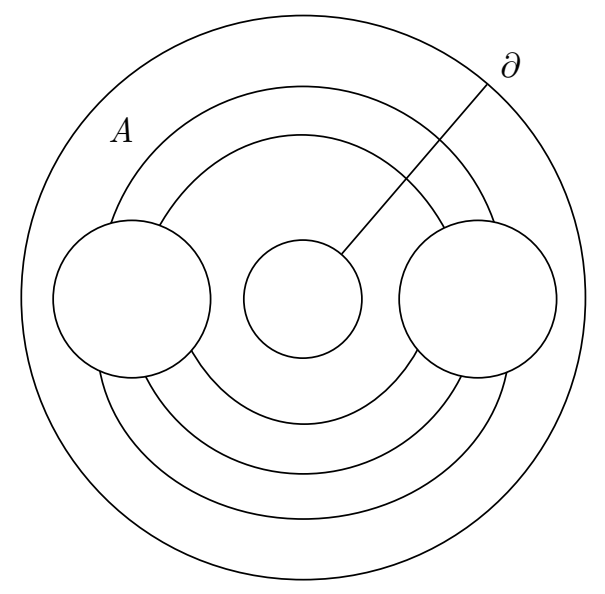

Figure 5

on $T$. Let $H$ be a 1-handle embedded in $M$ (i.e., $H$ is a tubular neighbourhood of a proper arc in $M)$ so that $H \cap \partial M=H \cap A$ consists of two attaching discs of $H$. Suppose that the arc of the core of $H$ is oriented. Let $Q$ be the annulus $\partial H$-int(attaching discs). Let $X$ be the exterior of $H$, that is, the closure of $M-H$. Let $P_{A}=A \cap X$. Let $D$ be an oriented disc properly embedded in $X$. We say $D$ is a non-trivial Scharlemann disc of $H$ if $\partial D \subset\left(P_{A} \cup Q\right)$ and $\partial D \cap Q$ consists of more than one arc on $Q$ such that orientations of these arcs coincide with that of the core of $H$. The number $\ell$ of the components of $\partial D \cap Q$ is called the multiplicity of $D$. As in Section 2, we define the Scharlemann co-cycle of $D$ to be the graph on $A$ whose vertices are fat vertices corresponding to the attaching discs of $H$ and whose edges are subarcs $\partial D \cap A$.

Lemma 3.2. If a Scharlemann co-cycle of a non-trivial Scharlemann disc $D$ is not contained in any disc on $A$, then $M$ does not contain a proper disc $E$ such that $\partial E \cap A$ consists of an essential arc on $A$.

Proof. We assume for a contradiction that such a disc $E$ exists. Since $\partial E \cap A$ consists of an essential arc on $A, E$ is non-separating in $M$. The Scharlemann co-cycle has two parallel families of edges. We can take the disc $E$ so that $\partial E \cap A$ consists of an arc which intersects precisely one parallel family of edges of the Scharlemann co-cycle in the minimal number of points, say $(0<) \ell^{\prime}(<\ell)$ points as shown in Figure 5.

Further we take $E$ so that $E$ intersects $H$ in discs which are parallel to the cocore disc of $H$. Each such disc intersects $\partial D$ in $\ell$ points. Let $w$ be the algebraic intersection number of $E$ and the core of $H$. Then the disc $E$ intersects $\partial D$ algebraically $\ell w \pm \ell^{\prime} \neq 0$ times. Hence the boundary loop $\partial D$ represents a non-zero element of $H_{1}(M ; \mathbb{Z})$, which is a contradiction.

We return to our original situation and prove Lemma 3.1.

Proof of Lemma 3.1. For the Scharlemann cycle $\sigma_{i}$ in $\Gamma_{D}$, we define the Scharlemann co-cycle of $\sigma_{i}$ as in the last paragraph in Sect.2. If at least one of the Scharlemann co-cycles of $\sigma_{1}$ and $\sigma_{2}$, say that of $\sigma_{1}$, is contained in a disc on $A$, then we perform surgery on $A$ along $\sigma_{1}$ and obtain a separating annulus $B$ with 


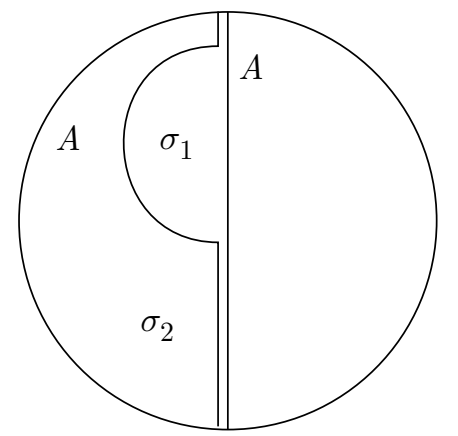

(1)

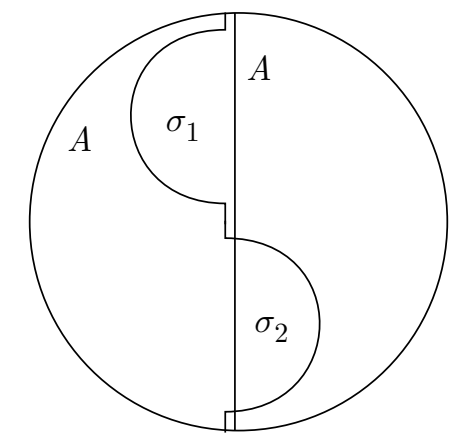

$(2)$

FigurE 6

$\partial B=\partial A$ and $\left|B \cap K^{*}\right|=\left|A \cap K^{*}\right|-2$, which is essential since $A$ is essential in $W(K ; \gamma)$. This contradicts the minimality of $q_{A}$. Hence we can assume that none of the Scharlemann co-cycles of $\sigma_{1}$ and $\sigma_{2}$ is contained in a disc on $A$.

Let $E_{1}$ and $E_{2}$ be the disc faces of $\sigma_{1}$ and $\sigma_{2}$. The separating annulus $A$ divides $W(K ; \gamma)$ into two 3 -manifolds $M_{1}$ and $M_{2}$.

Suppose first that both discs $E_{1}$ and $E_{2}$ are in the same component, say $M_{1}$. Then surgery on the annulus $A$ along the Scharlemann cycle $\sigma_{1}$ yields a separating annulus $A^{\prime}$ in $W(K ; \gamma)$ with $\partial A^{\prime}=\partial A$ and $\left|A^{\prime} \cap K^{*}\right|=\left|A \cap K^{*}\right|-2$. See Figure 6 (1). The annulus $A^{\prime}$ divides $W(K ; \gamma)$ into two 3 -manifolds $M_{1}^{\prime}$ and $M_{2}^{\prime}$, where $M_{1}^{\prime}$ contains the disc $E_{2}$. The annulus $A^{\prime}$ is incompressible since $A$ is incompressible and $\partial A^{\prime}=\partial A$. We show that $A^{\prime}$ is $\partial$-incompressible in $W(K ; \gamma)$. Clearly the annulus $A^{\prime}$ does not have a $\partial$-compressing disc in $M_{1}^{\prime}$, by Lemma 3.2. Let us regard a regular neighbourhood of $E_{1}$ as a 1-handle $H$, and the co-core of the 1-handle $(\subset V)$ between fat vertices numbered $x$ and $x+1$ in $\Gamma_{A}$ as a "Scharlemann disc" of $H$. Since we are assuming that the Scharlemann co-cycle of $\sigma_{1}$ is not contained in a disc on $A$, the Scharlemann co-cycle of $H$ is not contained in a disc in $A^{\prime}$. Hence $A^{\prime}$ does not have a $\partial$-compressing disc also in $M_{2}^{\prime}$, by Lemma 3.2. Thus $A^{\prime}$ is essential in $W(K ; \gamma)$, which contradicts the minimality of $q_{A}$.

Secondly we assume the discs $E_{1}$ and $E_{2}$ are in distinct components, say in $M_{1}$ and in $M_{2}$ respectively.

Case (a). If $\{x, x+1\} \cup\{y, y+1\}$ consists of four elements, then we perform two surgeries on the annulus $A$ simultaneously along the two Scharlemann cycles $\sigma_{1}$ and $\sigma_{2}$. This operation yields a separating annulus $A^{\prime \prime}$ in $W(K ; \gamma)$ with $\partial A^{\prime \prime}=\partial A$ and $\left|A^{\prime \prime} \cap K^{*}\right|=\left|A \cap K^{*}\right|-4$. See Figure 6 (2).

Case (b). If $\{x, x+1\} \cup\{y, y+1\}$ consists of three elements, then we isotope 1handles between $x$ and $x+1$ and between $y$ and $y+1$, the knot $K^{*}$ and $\operatorname{discs} E_{1}$ and $E_{2}$ as shown in Figure 7.

We perform a similar operation on $A$ as in Case (a), and obtain an annulus $A^{\prime \prime}$ such that $\left|A^{\prime \prime} \cap K^{*}\right|=\left|A \cap K^{*}\right|-2$.

In both cases (a) and (b), similar arguments as for the annulus $A^{\prime}$ show that $A^{\prime \prime}$ is essential in $W(K ; \gamma)$.

Case (c). If $\{x, x+1\} \cup\{y, y+1\}$ consists of two elements, then $q_{A}=2$. 


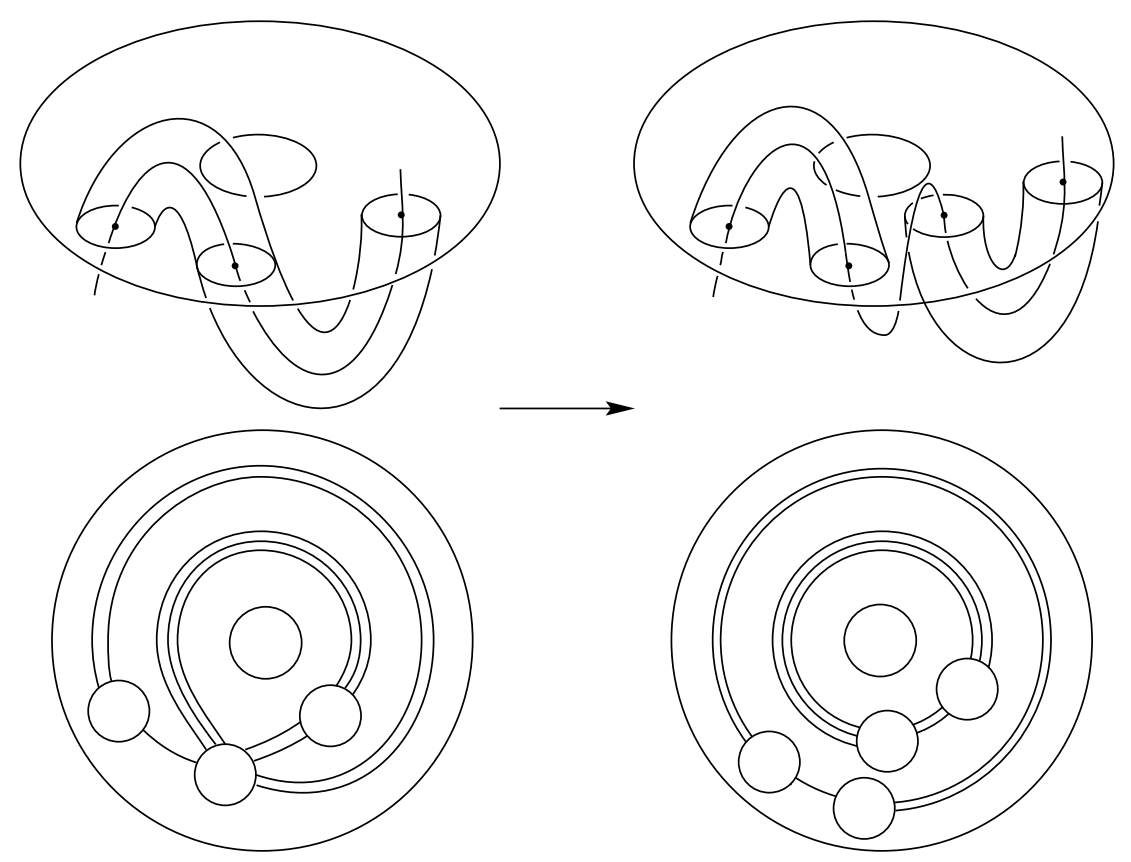

FiguRE 7

\section{LEMMAS ABOUT INTERIOR EDGES}

Lemma 4.1. ([15, Proposition 5.1]) If the graph $\Gamma_{i}$ contains a great $x$-edge cycle, then its disc face contains a disc face of a Scharlemann cycle.

Lemma 4.2. $\Gamma_{A}$ contains at most $q_{A}$ sign-preserving $x$-edges for every label $x$.

Proof. Suppose that $\Gamma_{A}$ contains $k$ sign-preserving $x$-edges. Let $\Lambda$ be the subgraph of $\Gamma_{A}$ consisting of the above sign-preserving $x$-edges and all the vertices of $\Gamma_{A}$. The graph $\Lambda$ may have an isolated vertex. Let $f_{d}$ denote the number of disc faces of $\Lambda$. Applying Euler's formula for the graph $\Lambda$ on $A$, we have:

$$
q_{A}-k+\Sigma \chi(\text { face })=\chi(A)=0 .
$$

Hence if $k \geq q_{A}+1$, then we have $f_{d} \geq \Sigma \chi($ face $) \geq 1$ and $\Gamma_{A}$ contains a great $x$-edge cycle. This contradicts Lemmas 4.1 and 2.3.

Lemma 4.3. $\Gamma_{A}$ contains at most $q_{D} q_{A} / 2$ sign-preserving edges.

Proof. Suppose that $\Gamma_{A}$ has more than $q_{D} q_{A} / 2$ sign-preserving edges. Their end points are more than $q_{D} q_{A}$. Since every sign-preserving edge has distinct labels at its two end points by the parity rule and since $\Gamma_{A}$ has $q_{D}$ kinds of labels at end points of interior edges, there are more than $q_{A}$ sign-preserving $x$-edges for some label $x$. Then the result follows by Lemma 4.2.

Lemma 4.4. If $\Gamma_{D}$ contains more than $q_{D}-1$ Scharlemann cycles, then $q_{A}=2$.

Proof. If $\Gamma_{D}$ contains Scharlemann cycles for at least two distinct intervals, then the result follows from Lemma 3.1. So we assume for a contradiction that $\Gamma_{D}$ has 


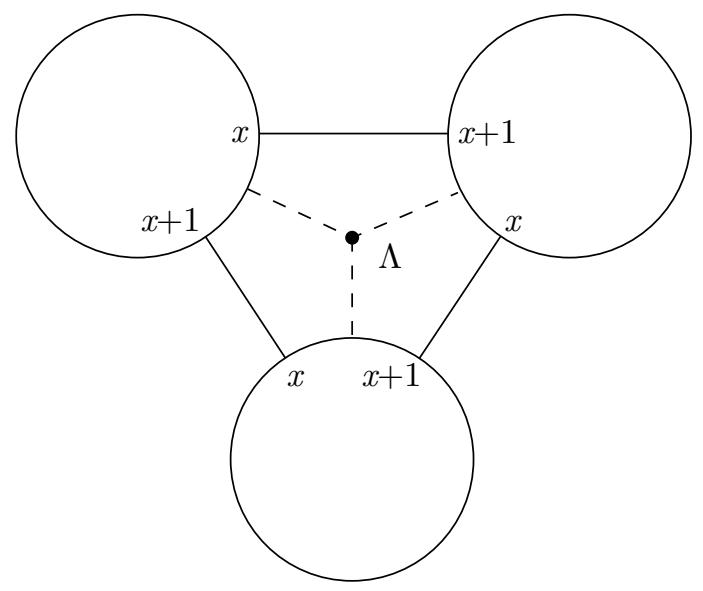

Figure 8

$k\left(\geq q_{D}\right)$ Scharlemann cycles, and they are for the same intervals, say $[x, x+1]$. The proof is contained in that of [13, Theorem 2.3].

We construct a graph $\Lambda$ in $D$ as follows. We choose a dual vertex in the interior of each disc face of $\Gamma_{D}$ bounded by a Scharlemann cycle for the interval $[x, x+1]$. Let the vertices of $\Lambda$ be the vertices of $\Gamma_{D}$ together with these dual vertices. The edges of $\Lambda$ are defined by joining each dual vertex to the vertices of the corresponding Scharlemann cycle in the obvious way. See Figure 8.

Since $\Gamma_{D}$ does not contain a trivial Scharlemann cycle, $\Lambda$ has at least $2 k$ edges. Hence Euler's formula for the graph $\Lambda$ on $D$ implies

$$
\left(q_{D}+k\right)-2 k+\Sigma \chi(\text { face }) \geq \chi(D)=1 .
$$

Since $k \geq q_{D}$, we have $\Sigma \chi($ face $) \geq 1$. In particular, there is a disc face $E$ of $\Lambda$. But $\partial E$ determines an $x$-edge cycle $\sigma$ bounding a disc in $E$. See Figure 9.

By Lemma 4.1 the disc face of $\sigma$ contains a Scharlemann cycle, which contradicts the way we constructed $\Lambda$.

Lemma 4.5. If $\Gamma_{D}$ contains more than $2 q_{D}-2$ sign-preserving $x$-edges for some label $x$, then $q_{A}=2$.

Proof. Suppose that $\Gamma_{D}$ contains $k$ sign-preserving $x$-edges. Let $\Lambda$ be the subgraph of $\Gamma_{D}$ consisting of the above sign-preserving $x$-edges and all the vertices of $\Gamma_{D}$. The graph $\Lambda$ may have an isolated vertex. Let $f_{d}$ denote the number of disc faces of $\Lambda$. Applying Euler's formula for the graph $\Lambda$ on $D$, we have

$$
q_{D}-k+\Sigma \chi(\text { face })=\chi(D)=1 .
$$

Hence if $k \geq 2 q_{D}-1$, then we have $f_{d} \geq \Sigma \chi($ face $) \geq q_{D}$ and $\Gamma_{A}$ contains at least $q_{D}$ great $x$-edge cycles whose disc faces are disjoint from each other. By Lemma $4.1 \Gamma_{D}$ contains at least $q_{D}$ Scharlemann cycles. Hence $q_{A}=2$ follows by Lemma 4.4 .

Lemma 4.6. If $\Gamma_{D}$ contains more than $\left(2 q_{D}-2\right) q_{A} / 2$ sign-preserving edges, then $q_{A}=2$. 


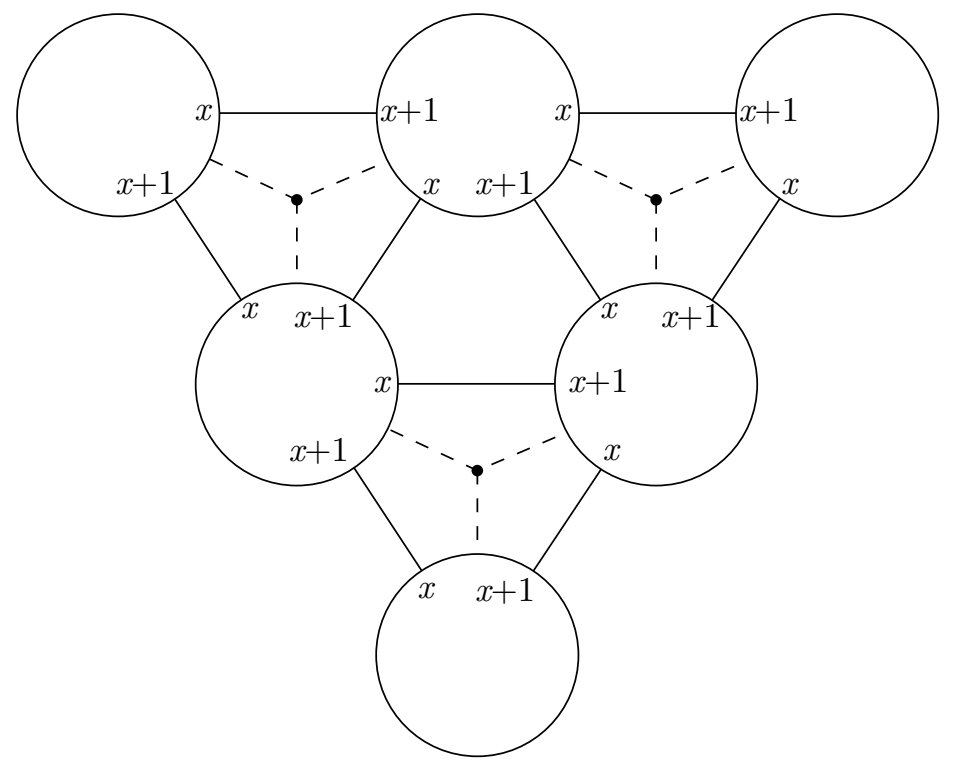

Figure 9

Proof. Suppose that $\Gamma_{D}$ has more than $\left(2 q_{D}-2\right) q_{A} / 2$ sign-preserving edges. Their end points are more than $\left(2 q_{D}-2\right) q_{A}$. Since every sign-preserving edge has distinct labels at its two end points by the parity rule and since $\Gamma_{D}$ has $q_{A}$ kinds of labels at end points of interior edges, there are more than $2 q_{D}-2$ sign-preserving $x$-edges for some label $x$. Then the result follows by Lemma 4.5.

The next Lemmas 4.7 and 4.8 are well-known, and we omit the proof of the former.

Lemma 4.7. ([2, Lemma 2.6.7]) The graph $\Gamma_{A}$ does not contain a parallel family of more than $q_{D} / 2$ sign-preserving edges. (Otherwise, $\Gamma_{A}$ would contain a Scharlemann cycle.)

Lemma 4.8. [10, Proposition 1.3]) If $\Gamma_{A}$ contains a parallel family of more than $q_{D}-1$ interior edges, then the knot $K$ is a cable knot in $W$.

Proof. First we note that $q_{D}>1$, for otherwise (i.e., $q_{D}=1$ ), $\Gamma_{D}$ contains a trivial loop. This is a contradiction.

Suppose that $\Gamma_{A}$ contains a parallel family of more than $q_{D}-1$ interior edges. From Lemma 4.7 we see that these edges are sign-reversing edges. Then the result follows because the same arguments in the proof of [10, Proposition 1.3].

\section{Proof of Theorem 1.2}

We prove Theorem 1.2 in this section.

Lemma 5.1. Suppose that each component of $\partial A$ intersects $\partial D$ in one point, and $\Delta \geq 3$. Then $q_{A}=2$.

Proof. The graph $\Gamma_{A}$ has at most two boundary edges. It has at most $q_{D} q_{A} / 2$ sign-preserving edges by Lemma 4.3. Each vertex of $\Gamma_{A}$ has $\Delta q_{D}$ end points of 

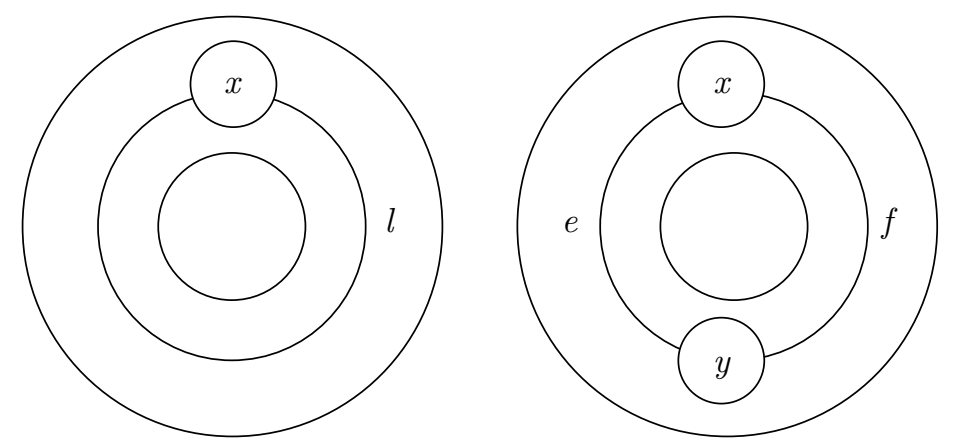

FIGURE 10

edges. Hence the number of end points of sign-reversing edges of $\Gamma_{A}$ is at least

$$
\begin{aligned}
3 q_{D} q_{A} & -2-\left(q_{D} q_{A} / 2\right) 2 \\
& =\left(2 q_{D}-2\right) q_{A}+2\left(q_{A}-1\right) \\
& >\left(2 q_{D}-2\right) q_{A} .
\end{aligned}
$$

Note that the last inequality follows because $q_{A} \geq 2$. Thus by the parity rule the graph $\Gamma_{D}$ has more than $\left(2 q_{D}-2\right) q_{A} / 2$ sign-preserving edges. Then $q_{A}=2$ follows by Lemma 4.6.

Lemma 5.2. Suppose that each component of $\partial A$ intersects $\partial D$ in one point, and $\Delta \geq 3$. Then the knot $K$ is a cable knot in $W$.

Proof. Since $\Gamma_{A}$ has at most two boundary edges, and since $\Gamma_{A}$ has precisely two vertices by Lemma $5.1, \Gamma_{A}$ has a vertex $x$ to which at most one boundary edge is incident. Let $y$ be the other vertex. Let $\widetilde{\Gamma}_{A}$ be the reduced graph corresponding to $\Gamma_{A}$, obtained by amalgamating all mutually parallel edges in the obvious way.

The graph $\widetilde{\Gamma}_{A}$ has at most one loop edge incident to the vertex $x$, say $\ell$. Otherwise, either the valencies of $x$ and $y$ would be different in $\Gamma_{A}$, or $\Gamma_{A}$ would contain a trivial loop. The graph $\widetilde{\Gamma}_{A}$ has at most two edges $e$ and $f$ connecting $x$ and $y$. See Figure 10. By Lemma $4.7, \ell$ corresponds to at most $q_{D} / 2$ parallel edges. Then the number of end points of edges corresponding to $e$ and $f$ at $x$ is at least

$$
3 q_{D}-1-\left(q_{D} / 2\right) 2=2\left(q_{D}-1\right)+1 .
$$

Thus $\Gamma_{A}$ contains a parallel family of more than $q_{D}-1$ interior edges connecting $x$ and $y$. Then by Lemma 4.8 the knot $K$ is a cable knot in $W$.

By Lemmas 5.1 and 5.2 we get the next proposition.

Proposition 5.3. Let $W, K, T, D$ and $A$ be as in Theorem 1.2. If $\Delta \geq 3$, then $K$ is a cable knot in $W$.

We can exclude the possibility of cable knots under the above condition by the next proposition, and hence we establish Theorem 1.2.

Proposition 5.4. Let $W, K, T, D$ and $A$ be as in Theorem 1.2. If $\Delta \geq 3$, then $K$ cannot be cabled in $W$. 
We need the next claim.

Claim 1. Let $K$ be a $(p, q)$-torus knot in a solid torus $M$ (i.e., $K$ is a $(p, q)$-cable of a core of $M)$ with $q \geq 2$. Let $\gamma$ be a slope on the torus $\partial N(K)$. If $M(K ; \gamma)$ contains a separating essential annulus $A$, then we can take another separating essential annulus $A^{\prime}$ in $M(K ; \gamma)$ such that $A^{\prime} \cap K^{*}=\emptyset$ and $\partial A^{\prime}=\partial A$.

Proof of Claim 1. Among all the separating essential annuli in $M(K ; \gamma)$ such that their boundary coincides with $\partial A$, we choose an annulus $A^{\prime}$ so that $\left|A^{\prime} \cap K^{*}\right|$ is minimal. For a contradiction, assume $A^{\prime} \cap K^{*} \neq \emptyset$. Then $A^{\prime} \cap\left(M(K ; \gamma)-\operatorname{int} N\left(K^{*}\right)\right)$ is an essential punctured annulus in the cable space

$$
M-\operatorname{Int} N(K)=M(K ; \gamma)-\operatorname{Int} N\left(K^{*}\right) .
$$

By [10, Lemma 3.1], this essential punctured annulus does not separate the cable space, and hence $A^{\prime}$ is also non-separating in $M(K ; \gamma)$. This is a contradiction.

Let $K$ be a cable knot of a knot $J$ (possibly a core of $W$ ) in $W$. Suppose for a contradiction that for a slope $\gamma$ with $\Delta \geq 3$ the 3 -manifold $W(K ; \gamma)$ contains a separating essential annulus $A$ such that every component of $\partial A$ intersects $\partial D$ once. We assume that $\left|A \cap K^{*}\right|$ is minimal. The exterior $W-\operatorname{Int} N(K)$ contains a cable space $C$ such that $\partial N(K) \subset C$. The union $M=C \cup N(K)$ is a solid torus.

Claim 2. The torus $\partial M$ is incompressible in the exterior $W-\operatorname{int} N(K)$.

Proof of Claim 2. Clearly $\partial M$ is incompressible in $C$. Suppose for a contradiction that $\partial M$ has a compressing disc $E$ in the closure of $W-M$. Let $B$ be a regular neighbourhood of $M \cup E$. Then $\partial B$ is a separating 2-sphere in $W$. By a standard cut and paste argument we can rechoose the compressing disc $D$ of $T$ so that $D \cap \partial B=\emptyset$, which implies $D \cap K=\emptyset$. This contradicts the assumption that $T$ is incompressible in $W-\operatorname{int} N(K)$.

Since $\Delta \geq 2$, [7, Lemma 7.2$]$ implies that the manifold $M(K ; \gamma)$ is either

(1) a solid torus, or

(2) a Seifert fibred manifold over a disc with two exceptional fibres.

In the latter case (2), by a standard cut and paste argument we can rechoose an essential annulus $A$ so that $A \cap \partial M(K ; \gamma)$ consists of loops which are essential on $A$ and $\partial M(K ; \gamma)$, and so that $A \cap M(K ; \gamma)$ is empty or consists of separating essential annuli $A_{1}, \ldots, A_{k}$ in $M(K ; \gamma)$. Let $A_{\ell}^{\prime}$ be a separating essential annulus in $M(K ; \gamma)$ with $\partial A_{\ell}^{\prime}=\partial A_{\ell}$ and such that $A_{\ell}^{\prime} \cap K^{*}=\emptyset$ for $1 \leq \ell \leq k$. Such annuli exist by Claim 1. Replace $A_{1}, \ldots, A_{k}$ by $A_{1}^{\prime}, \ldots A_{k}^{\prime}$. Thus we can rechoose $A$ so that $A \cap K^{*}=\emptyset$, which is impossible by Lemma 2.1 .

In the former case (1), it is sufficient to prove this proposition for the corresponding Dehn surgery on the knot $J$ in $W$. Note that the distance $\Delta\left(\mu_{J}, \gamma_{J}\right) \geq 4$ by [7, Lemma 3.3], where $\mu_{J}$ and $\gamma_{J}$ are meridian slopes on the boundary of the solid tori $M$ and $M(K ; \gamma)$ respectively. Hence by Proposition $5.3 J$ is a cable knot in $W$. In particular we assume $\partial M$ and $T$ are not parallel. Then $\partial M$ is an essential torus in $W-\operatorname{int} N(K)$ by Claim 2. Thus Haken's finiteness theorem (see [17, III.20. Theorem]) and an inductive argument allow us to assume that (2) occurs rather than (1). 


\section{Surgeries CREATING NON-SEPARATING ANNULI}

In this section we prove Theorem $1.3(2)$.

Proof of Theorem 1.3 (2). By twisting $(-p)$-times along the meridian disc $D$, we get a new knot $K_{-p} \subset W \subset S^{3}$. Then we can easily compute that the surgery slope $m / n$ on $\partial N(K)$ corresponds to $\left(m-n p w^{2}\right) / n$ on $\partial N\left(K_{-p}\right)$, where $w$ denotes the algebraic intersection number of $K$ and $D$. Hence $W\left(K_{-p} ;\left(m-n p w^{2}\right) / n\right)$ contains a non-separating anulus $A^{\prime}$ whose boundary component is represented by a preferred longitude $L$. Let $W^{\prime}$ be the complementary solid torus $S^{3}-\operatorname{int} W$. Then $S^{3}\left(K_{-p} ;\left(m-n p w^{2}\right) / n\right)=W\left(K_{-p} ;\left(m-n p w^{2}\right) / n\right) \cup W^{\prime}$. Since $L$ bounds a meridian disc of $W^{\prime}$, we have a non-separating 2-sphere by capping off $\partial A^{\prime}$ with two meridian discs of $W^{\prime}$. Therefore [4, Corollary 8.3] implies that $K_{-p}$ is a trivial knot in $S^{3}$ and $\left(m-n p w^{2}\right) / n=0$, and hence $m / n=p w^{2}$. This completes the proof.

\section{LEMMAS ABOUT BOUNDARY EDGES}

To prove Theorem 1.1 we need more lemmas concerning boundary edges.

Let $b$ be a boundary edge. Suppose that $b$ is incident to vertices $x$ and $y$ in the graphs $\Gamma_{D}$ and $\Gamma_{A}$ respectively. We define the character of $b$ as char $b=$ $(\operatorname{sign} x) \times(\operatorname{sign} y)$.

Lemma 7.1. Let $e$ and $f$ be boundary edges. Then the edges $e$ and $f$ are incident to the same component of $\partial A$ if and only If char $e=\operatorname{char} f$.

Proof. The boundary loop $\partial D$ intersects the two boundary loops of $\partial A$ alternately. Suppose that $e$ and $f$ are boundary edges such that they have end points adjacent on $\partial D$. It is sufficient to prove that char $e=-\operatorname{char} f$.

Let $\alpha$ be the subarc of $\partial D$ connecting end points of $e$ and $f$ such that $\alpha$ does not meet any other end points of boundary edges. Let $F$ be the face of the graph $\Gamma_{D}$ whose closure contains $e \cup f \cup \alpha$, and let $x$ and $y$ be corners adjacent to $e$ and $f$ on the limit curves of the end of the open collar of $F$. See Figure 11.

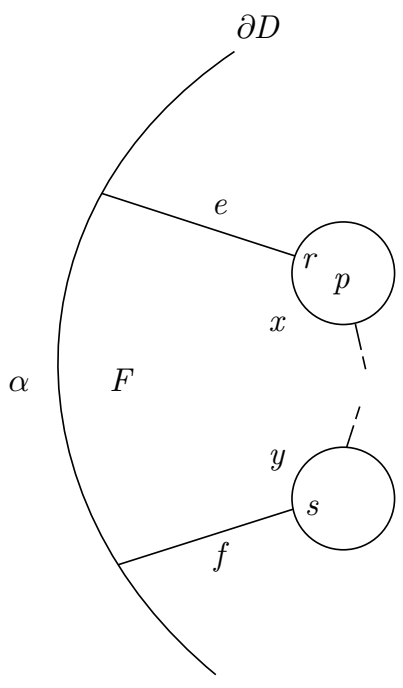

Figure 11 


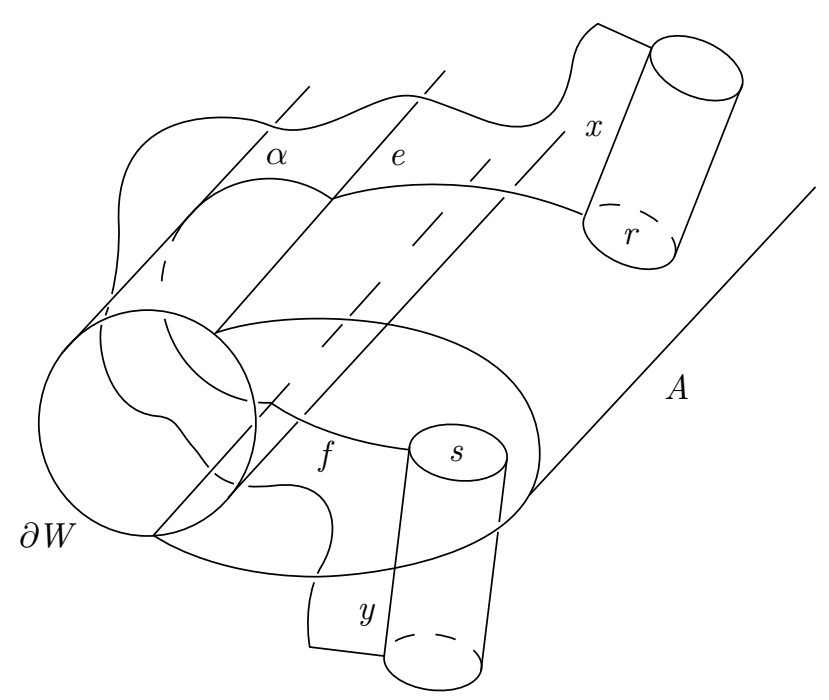

FigURE 12

Let $p, q$ be the vertices to which $e, f$ are incident in $\Gamma_{D}$, and $r, s$ the labels at the corresponding end points of $e, f$ respectively. Let $t$ and $u$ be subarcs of $K^{*}$ corresponding to $x$ and $y$, respectively. These four arcs $t, u, x$ and $y$ are in the normal direction to the annulus $A$ and have end points at the vertices $r$ or $s$. Because $\partial D$ and $\partial A$ are contained in the torus $T$ and $\alpha$ connects distinct components of $\partial A, \alpha$ connects the same side of $A$. Then the $\operatorname{arcs} x$ and $y$ have mutually inverse orientations with respect to $A$ near the vertices $r$ and $s$ when they are assigned the orientations induced from that of the face $F$. See Figure 12.

Hence the orientations of $t$ and $u$ induced by that of $K^{*}$ coincide with respect to $A$ near the vertices $r$ and $s$ if and only if the vertices $p$ and $q$ have mutually inverse signs. Thus the signs of the vertices $r$ and $s$ coincide if and only if the vertices $p$ and $q$ have mutually inverse signs.

The next Lemma 7.2 is a corollary of Lemma 7.1.

Lemma 7.2. Let $e$ and $f$ be a pair of parallel boundary edges of $\Gamma_{A}$. Let $r$ and $s$ be the labels of $e$ and $f$ at the vertex of $\Gamma_{A}$. Then the vertices numbered $r$ and $s$ in the graph $\Gamma_{D}$ have the same sign.

Let $\widetilde{\Gamma}_{A}$ be the reduced graph corresponding to $\Gamma_{A}$, obtained by amalgamating all mutually parallel edges in the obvious way.

Lemma 7.3. Let $\Lambda$ be a subgraph of $\widetilde{\Gamma}_{A}$, and $q$ the number of vertices of $\Lambda$. Then the graph $\Lambda$ has at most $2 q$ boundary edges. Moreover if it has precisely $2 q$ boundary edges, then it has at most $q$ interior edges.

Proof. Let $\Lambda^{\prime}$ be the subgraph of $\Lambda$ consisting of vertices and boundary edges of $\Lambda$. Let $v, e$ and $f$ be the numbers of vertices, edges and faces of $\Lambda^{\prime}$. Adding extra boundary edges if necessary, we may assume that $\Lambda^{\prime}$ has the maximal number of boundary edges. That is, we assume that adding another boundary edge to $\Lambda^{\prime}$ creates a parallel pair of boundary edges. Then the annulus $A$ is divided by these boundary edges into discs each of which is bounded by a 4 -gon whose corners are 
in $\partial A$ and in a fat vertex alternately. Hence $2 e=4 f$, and Euler's formula implies that $0=v-e+f=q-(e / 2)$. Thus we have $e=2 q$, and hence $\Lambda^{\prime}$ has at most $2 q$ boundary edges. Moreover if $\Lambda$ has exactly $2 q$ boundary edges, then $\Lambda^{\prime}$ has also $2 q$ boundary edges, and $f=e-v=2 q-q=q$. Since $\Lambda$ has at most one interior edge in every face of $\Lambda^{\prime}, \Lambda$ has at most $q$ interior edges.

Lemma 7.4. Let $\Lambda$ be a subgraph of $\widetilde{\Gamma}_{A}$, and $q$ the number of vertices of $\Lambda$. Then for each component $C$ of $\partial A$ the graph $\Lambda$ has at most $2 q-1$ boundary edges incident to $C$.

Proof. Let $\Lambda^{\prime}$ be the graph consisting of all vertices of $\Lambda$ and boundary edges of $\Lambda$ which are incident to $C$. Let $v, e$ and $f$ be the numbers of vertices, edges and faces of $\Lambda^{\prime}$. We assume that $\Lambda^{\prime}$ has the maximal number of boundary edges incident to $C$. Then the annulus $A$ is divided by these boundary edges into a once punctured disc bounded by a bi-gon and discs bounded by 4-gons. Hence $2 e=4(f-1)+2$, and Euler's formula implies that $0=v-e+(f-1)=q-e+(e-1) / 2$. Thus we have $e=2 q-1$, and $\Lambda$ has at most $2 q-1$ boundary edges incident to $C$.

Lemma 7.5. Assume that $q_{D} \geq 2$. If $\Gamma_{A}$ contains a parallel family of more than $2 q_{D}-2$ boundary edges, then the knots $K$ and $K^{*}$ are parallel to essential simple loops on $T$ in $W$ and $W(K ; \gamma)$ respectively.

Proof. In the graph $\Gamma_{D}$, every vertex (except possibly for one) has two boundary edges incident to it, corresponding to $2 q_{D}-1$ parallel successive boundary edges in $\Gamma_{A}$. Hence there are parallel edges $e$ and $f$ in $\Gamma_{D}$ among these boundary edges. Let $x$ and $y$ be the vertices of $\Gamma_{A}$ and $\Gamma_{D}$ to which $e$ and $f$ are incident. Let $Q_{i} \subset P_{i}$ be the disc of parallelism of the edges $e$ and $f$ in the graph $\Gamma_{i}$ for $i=D$ and $A$. We can isotope the annulus $Q_{D} \cup Q_{A}$ slightly so that its boundary intersects the loop $\partial y$ in one point. Recall that $\partial y$ is a meridian loop of the knot $K$. Thus the knot $K$ is parallel to an essential simple loop on $T$ in $W$.

If $Q_{D}$ does not contain a boundary edge with label $x$ except for $e$ and $f$, then we can isotope the annulus $Q_{D} \cup Q_{A}$ slightly so that its boundary intersects the loop $\partial x$ in one point.

If $Q_{D}$ contains such a boundary edge, then $Q_{D}$ contains a parallel family $B$ of $2 q_{A}$ boundary edges. The boundary edges of $\Gamma_{A}$ corresponding to them are incident to a component, say $C$, of $\partial A$ if they are incident to vertices of $\Gamma_{A}$ with the sign + , and to the other component $C^{\prime}$ of $\partial A$ if they are incident to vertices of $\Gamma_{A}$ with the sign - by Lemma 7.1. Let $\Lambda$ be the subgraph of $\Gamma_{A}$ consisting of the vertices with the sign + and boundary edges incident to these vertices and corresponding to those of $B$. Since every vertex of $\Lambda$ has two boundary edges incident to it, $\Lambda$ has parallel boundary edges by Lemma 7.4. These two edges are parallel also in $\Gamma_{A}$, and similar arguments as in the first paragraph of this proof show that $K^{*}$ is parallel to an essential simple loop on $T$ in $W(K ; \gamma)$.

Lemma 7.6. If $q_{D}=1$ and $\Delta \geq 2$, then the knots $K$ and $K^{*}$ are parallel to essential simple loops on $T$ in $W$ and $W(K ; \gamma)$ respectively.

Proof. Since $\Gamma_{D}$ has precisely one vertex $x$, it does not have interior edges, and has $\Delta q_{A} \geq 2 q_{A}$ parallel boundary edges. Then $\Gamma_{A}$ also does not have interior edges, and it has parallel boundary edges $e$ and $f$ incident to a vertex, say $y$, such that $e$ and $f$ cobound a disc of parallelism $Q_{A}\left(\subset P_{A}\right)$. We may assume that $Q_{A}$ does not contain boundary edges other than $e$ and $f$. Let $Q_{D}\left(\subset P_{D}\right)$ be the disc of 
parallelism of the edges $e$ and $f$. Then the annulus $Q_{A} \cup Q_{D}$ gives a parallelism of $K$ and a simple loop on $T$ in $W$.

Since $\Gamma_{D}$ has a parallel family $B$ of $2 q_{A}$ boundary edges, the same argument as in the last paragraph in the proof of Lemma 7.5 shows that $K^{*}$ is parallel to a simple loop on $T$ in $W(K ; \gamma)$.

In the following we assume $q_{D}>1$.

\section{Proof of Theorem 1.1 in Case $q_{A}=2$}

The goal of this section is to prove the following proposition, which guarantees Theorem 1.1 for $\Delta \geq 3$ when $q_{A}=2$. Lemmas $8.2,8.3,8.4$ and 8.5 form a proof of this proposition.

Proposition 8.1. Assume that $q_{A}=2$. If $\Delta \geq 3$, then either (1) the knot $K$ is a cable knot in $W$, or (2) the knots $K$ and $K^{*}$ are parallel to essential simple loops on $T$ in $W$ and $W(K ; \gamma)$ respectively.

Let $p_{D}$ and $m_{D}$ be the numbers of vertices of $\Gamma_{D}$ with sign + and - respectively. We assume without loss of generality that $p_{D} \geq m_{D}$.

Lemma 8.2. Assume that $q_{A}=2$. Suppose that one of the two vertices of $\widetilde{\Gamma}_{A}$, say $x_{1}$, has two boundary edges which are incident to $x_{1}$ and to distinct components of $\partial A$. If $\Delta \geq 3$, then the knot $K$ is a cable knot in $W$.

Proof. Let $C_{1}$ and $C_{2}$ be the two components of $\partial A$. The graph $\widetilde{\Gamma}_{A}$ consists of two vertices $x_{1}$ and $x_{2}$. Let $b_{s t}$ be a boundary edge connecting $x_{s}$ and $C_{t}$ for $s, t=1,2$. Let $e$ and $f$ be interior edges connecting $x_{1}$ and $x_{2}$. Let $\ell$ be a loop edge incident to $x_{1}$. Note that $b_{21}, b_{22}, e, f$ and $\ell$ may not exist. See Figure 13.

We assume without loss of generality that in the graph $\Gamma_{A}$ the labels at the end points of the edges corresponding to $b_{11}$ have the sign + . Then Lemma 7.2 implies that each of $b_{11}$ and $b_{22}$ corresponds to at most $p_{D}$ parallel boundary edges of $\Gamma_{A}$, and that each of $b_{12}$ and $b_{21}$ corresponds to at most $m_{D}$ parallel boundary edges of $\Gamma_{A}$. We consider the number of end points of edges at $x_{2}$ in $\Gamma_{A}$, and see that the sum of the numbers of end points of edges corresponding to $e$ and $f$ is at least

$$
3 q_{D}-\left(p_{D}+m_{D}\right)=2 q_{D} .
$$
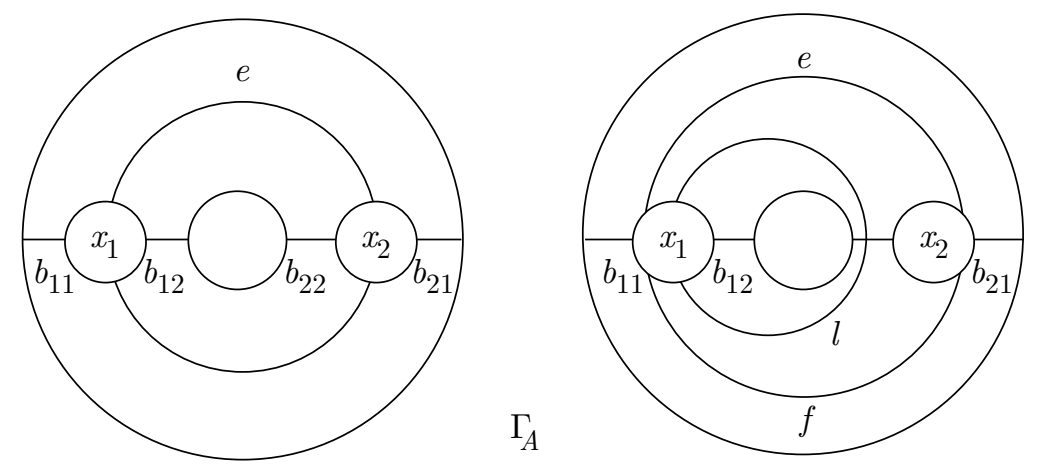

FiguRE 13 


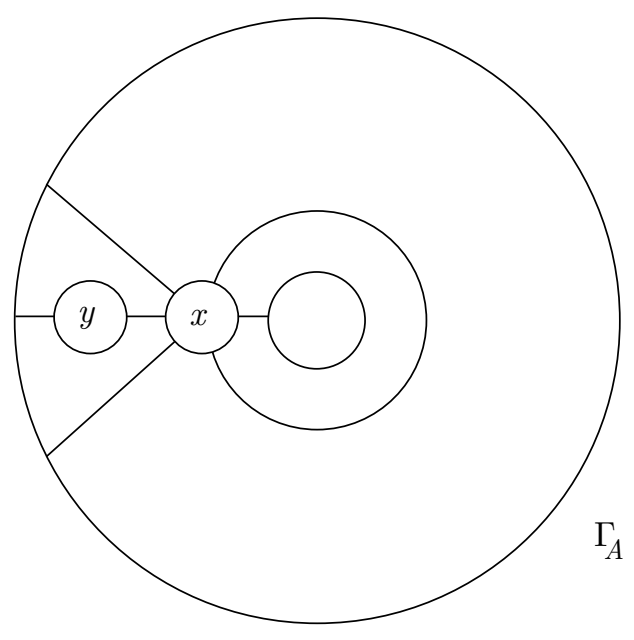

FiguRe 14

Then $e$ or $f$ corresponds to more than $q_{D}-1$ parallel interior edges, and the knot $K$ is a cable knot in $W$ by Lemma 4.8 .

Lemma 8.3. Assume that $q_{A}=2$ and that some vertex $x$ of $\widetilde{\Gamma}_{A}$ has two boundary edges incident to $x$ and the same component $C$ of $\partial A$. If $\Delta \geq 3$, then either (1) the knot $K$ is a cable knot in $W$ or (2) the knots $K$ and $K^{*}$ are parallel to essential simple loops on $T$ in $W$ and $W(K ; \gamma)$ respectively.

Proof. The other vertex $y$ of $\widetilde{\Gamma}_{A}$ has precisely one boundary edge $b$ and precisely one interior edge $e$ incident to it. See Figure 14.

Then since the vertex $y$ has valency at least $3 q_{D}$, either $b$ corresponds to more than $2 q_{D}-2$ parallel edges, or $e$ corresponds to more than $q_{D}-1$ parallel edges. In the former case we have (2) by Lemma 7.5. In the latter case we have (1) by Lemma 4.8 .

By Lemmas 8.2 and 8.3 we can assume that $\widetilde{\Gamma}_{A}$ is as in Figure 15 for the rest of this section.

Lemma 8.4. Assume that $q_{A}=2$ and that $\widetilde{\Gamma}_{A}$ is as shown in Figure 15. If $m_{D}>0$ and $\Delta \geq 3$, then the knot $K$ is a cable knot in $W$.

Proof. Let $x$ be a vertex of $\widetilde{\Gamma}_{A}$. The graph $\widetilde{\Gamma}_{A}$ has at most one loop edge and exactly one boundary edge incident to the vertex $x$, say $\ell$ and $b$ respectively. By Lemma 7.2 and the fact that $m_{D}>0, b$ corresponds to at most $p_{D}$ parallel boundary edges of $\Gamma_{A}$. If the sum of the numbers of end points of $\ell$ and $b$ at $x$ is more than $q_{D}$, then we find two edges with the same label, say $r$, in parallel edges of $\Gamma_{A}$ corresponding to $\ell$, and they form a great $r$-edge cycle, which is a contradiction by Lemmas 2.3 and 4.1. The graph $\widetilde{\Gamma}_{A}$ has at most two edges $e$ and $f$ connecting the two vertices of $\widetilde{\Gamma}_{A}$. The number of end points of edges of $\Gamma_{A}$ corresponding to $e$ and $f$ at $x$ sums up to at least $3 q_{D}-q_{D}=2 q_{D}$. Thus $\Gamma_{A}$ contains a parallel family of more than $q_{D}-1$ interior edges connecting the two vertices of $\Gamma_{A}$. Then by Lemma 4.8 the knot $K$ is a cable knot in $W$. 


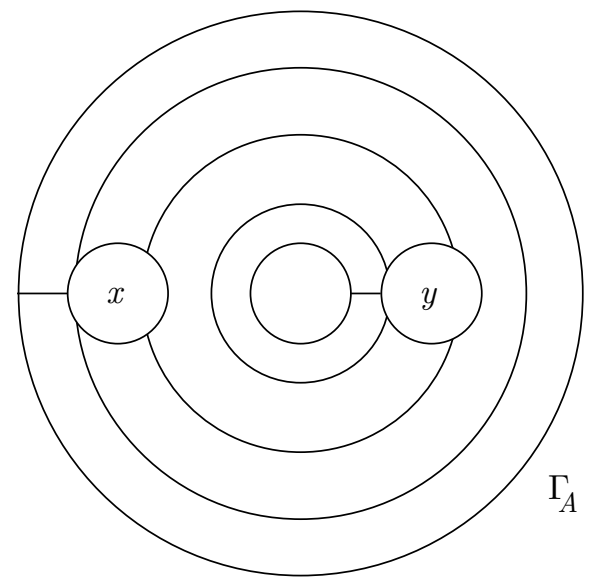

FiguRe 15

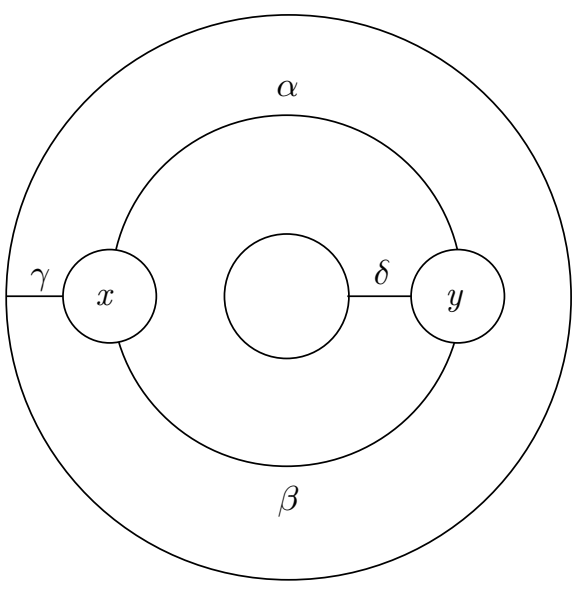

FiguRe 16

Lemma 8.5. Assume that $q_{A}=2$ and that $\widetilde{\Gamma}_{A}$ is as shown in Figure 15. If $m_{D}=0$ and $\Delta \geq 3$, then either (1) the knot $K$ is a cable knot in $W$ or (2) the knots $K$ and $K^{*}$ are parallel to essential simple loops on $T$ in $W$ and $W(K ; \gamma)$ respectively.

Proof. Since $m_{D}=0$, the graph $\Gamma_{D}$ has no sign-reversing edges, and hence $\Gamma_{A}$ has no sign-preserving edges by the parity rule. Hence $\widetilde{\Gamma}_{A}$ has no loop edges, as shown in Figure 16.

Sign-reversing edges $\alpha$ and $\beta$ of $\widetilde{\Gamma}_{A}$ connect the two vertices $x$ and $y$ of $\widetilde{\Gamma}_{A}$. If $\alpha$ or $\beta$ corresponds to more than $q_{D}-1$ parallel edges of $\Gamma_{A}$, then (1) follows by Lemma 4.8. Hence we assume in the following that each of $\alpha$ and $\beta$ corresponds to at most $q_{D}-1$ parallel edges. The graph $\widetilde{\Gamma}_{A}$ has two boundary edges $\gamma$ and $\delta$ incident to $x$ and $y$ respectively. Since $\Delta \geq 3$ and each of $\alpha$ and $\beta$ corresponds to at most $q_{D}-1$ parallel edges, each of $\gamma$ and $\delta$ corresponds to at least $q_{D}+2$ parallel edges of $\Gamma_{A}$. At end points of each family of the parallel edges, we see some labels appear more than once. Thus every vertex of $\Gamma_{D}$ has at least two boundary edges 
incident to it, and there is a vertex with more than two boundary edges incident to it.

Claim 1. If $\Gamma_{D}$ has a parallel family of three boundary edges, then (2) follows.

Proof. Let $e, f$ and $g$ be three parallel boundary edges in $\Gamma_{D}$, placed in this order. Let $v$ be the vertex of $\Gamma_{D}$ which they are incident to. The edges $e$ and $g$ have the same label, say $x$, at $v$ since $q_{A}=2$. The vertex $x$ of $\widetilde{\Gamma}_{A}$ has exactly one boundary edge incident to it, because $\widetilde{\Gamma}_{A}$ is as shown in Figure 16. Hence the edges of $\Gamma_{A}$ corresponding to $e$ and $g$ (we will call them $e$ and $g$ in the following) are parallel in $\Gamma_{A}$. Let $Q\left(\subset P_{A}\right)$ be the disc of parallelism of these edges. If $Q$ contains an edge with the label $v$ other than $e$ and $g$, then $\Gamma_{A}$ has a parallel family of at least $2 q_{D}+1$ boundary edges, and we have (2) by Lemma 7.5. If $Q$ does not contain such an edge, then let $R$ be the disc of parallelism of $e$ and $g$ in $\Gamma_{D}$. We join the discs $Q$ and $R$ together along the $\operatorname{arcs} e$ and $g$, and obtain an annulus $B$ in the exterior $X$ of the knot $K$. By an adequate small isotopy of $B$, we have $|\partial B \cap \partial v|=1=|\partial B \cap \partial x|$. Thus (2) follows. This completes the proof of Claim 1.

Claim 2. Suppose that $\Gamma_{D}$ contains a Scharlemann cycle $\sigma$. If all the edges of $\sigma$ except for at most one edge have edges parallel to them, then (1) follows.

Proof. Let $e_{1}, e_{2}, \ldots, e_{n}$ be the edges of $\sigma$ placed in this order. Let $f_{k}$ be the edge which is adjacent and parallel to $e_{k}$ for $k=2, \ldots, n$. Let $v_{k}$ and $v_{k+1}$ be the vertices to which the edge $e_{k}$ is incident, where $v_{n+1}=v_{1}$. Note that every pair of parallel edges $e_{k}$ and $f_{k}$ forms a Scharlemann cycle $\sigma_{k}$ for $2 \leq k \leq n$, because $q_{A}=2$. The Scharlemann co-cycle of $\sigma_{k}$ is not contained in a disc on $A$ (otherwise we perform surgery on $A$ along $\sigma_{k}$ and have a contradiction to the minimality of $q_{A}$ ). We assume without loss of generality that the edge corresponding to $e_{1}$ is contained in the parallel family of edges of $\Gamma_{A}$ corresponding to $\alpha$. We indicate such a situation by writing $e_{1} \in \alpha$ in this proof. First suppose for a contradiction that $f_{k} \in \beta$ for $2 \leq k \leq n$. Then $e_{k} \in \alpha$ for $1 \leq k \leq n$, and the Scharlemann co-cycle of $\sigma$ is contained in a disc on $A$. Hence we perform surgery on $A$ along $\sigma$ and have a contradiction to the minimality of $q_{A}$. Second, we suppose that $f_{m} \in \alpha$ for some $m$. Assume that $m$ is the minimal integer as above. Then the edges $e_{m-1}$ and $f_{m}$ have the same label, say $x$, at the vertex $v_{m}$ since $q_{A}=2$. This implies that the family of parallel edges of $\Gamma_{A}$ corresponding to $\alpha$ contains two edges with the same label $v_{m}$ at the vertex $x$, and $\alpha$ corresponds to at least $q_{D}+1$ parallel edges of $\Gamma_{A}$. Thus (1) holds by Lemma 4.8. This completes the proof of Claim 2.

The next Claim 3 is an easy corollary of Claim 2.

Claim 3. If $\Gamma_{D}$ has a parallel family of more than two sign-preserving edges, then (1) follows.

We return to the main course of the proof of Lemma 8.5.

Since $\Gamma_{D}$ has a vertex with more than two boundary edges incident to it, we can assume by Claim 1 that $\Gamma_{D}$ has a vertex with a non-parallel pair of boundary edges incident to it. Let $e$ and $f$ be an outermost pair of such boundary edges, $Q$ the outermost disc on $D$, and $v$ the vertex of $\Gamma_{D}$ to which $e$ and $f$ are incident. By Claim 1 we can assume that every vertex on $Q$ other than $v$ has precisely two boundary edges which are parallel. We construct a new graph $\Lambda$ on $Q$ by moving the vertices on $Q$ other than $v$ to the boundary $\partial Q$ along the pairs of parallel boundary 


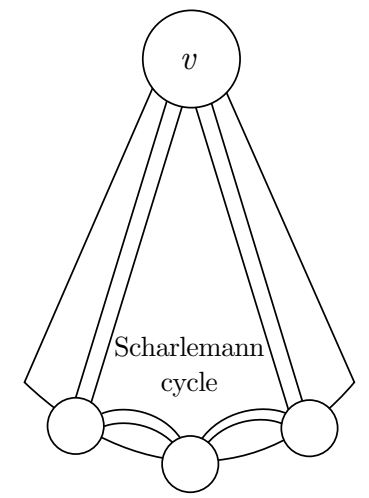

(a)

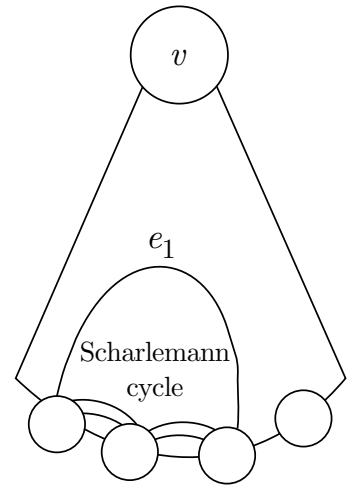

(b)

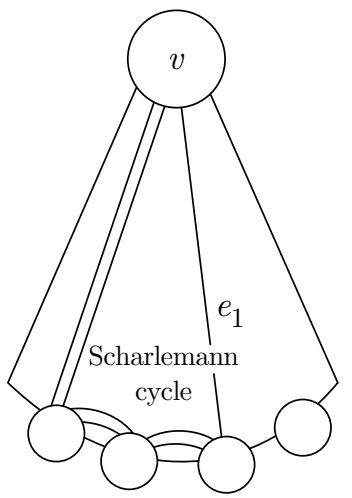

(c)

Figure 17

edges. We can assume that $\Lambda$ does not contain a parallel family of more than two edges by Claim 3, noting that all the interior edges of $\Gamma_{D}$ are sign-preserving because $m_{D}=0$. We call an edge $e$ of $\Lambda$ a diagonal edge if it connects two vertices which are not adjacent on $\partial Q$. Suppose first that $\Lambda$ does not have a diagonal edge. Then all the pairs of adjacent vertices are connected by two parallel edges. See Figure 17 (a). Every disc face of $\Lambda$ corresponds to a face of a Scharlemann cycle since $q_{A}=2$ and $m_{D}=0$. Hence (1) follows by Claim 2 .

Suppose second that $\Lambda$ has a diagonal edge. Let $e_{1}$ be an outermost diagonal edge on $Q, Q_{1}$ the outermost disc, and $v_{1}$ and $v_{2}$ the vertices to which $e_{1}$ is incident. Then every pair of adjacent vertices other than the pair $v_{1}$ and $v_{2}$ are connected by two parallel edges. See Figure 17 (b) and (c). We again have (1) by Claim 2.

\section{Proof of Theorem 1.1 when $\Gamma_{A}$ Does not contain A NON-PARALLEL PAIR OF DOUBLE BOUNDARY EDGES}

In this section we prove Theorem 1.1 when the graph $\Gamma_{A}$ does not contain a pair of non-parallel boundary edges which are incident to the same vertex and to the same component of $\partial A$. We call such a pair of boundary edges a non-parallel pair of double boundary edges. Proposition 8.1 and Lemmas 7.6, 9.1 and 9.2 form a proof of Theorem 1.1 for this situation.

Lemma 9.1. Assume that $q_{D} \geq 2$. Suppose that $\Gamma_{A}$ does not have a non-parallel pair of double boundary edges, and $m_{D}=0$. If $\Delta \geq 4$, then either (1) $q_{A}=2$, or (2) the knots $K$ and $K^{*}$ are parallel to essential simple loops on $T$ in $W$ and $W(K ; \gamma)$ respectively.

Proof. We assume that (2) does not hold to show that (1) holds. Then $q_{D} \geq 2$ and Lemma 7.5 imply that every boundary edge of $\widetilde{\Gamma}_{A}$ corresponds to at most $2 q_{D}-2$ parallel boundary edges. Since we assume that $\Gamma_{A}$ does not have a non-parallel pair of double boundary edges, every vertex of $\widetilde{\Gamma}_{A}$ has at most one boundary edge incident to it, by Lemma 7.2 and the fact that $m_{D}=0$. Hence $\Gamma_{A}$ has at most $\left(2 q_{D}-2\right) q_{A}$ boundary edges. Thus the number of end points of interior edges of 
$\Gamma_{A}$ is at least

$$
4 q_{D} q_{A}-\left(2 q_{D}-2\right) q_{A}=\left(2 q_{D}-2\right) q_{A}+4 q_{A} .
$$

Since $m_{D}=0, \Gamma_{D}$ has more than $\left(2 q_{D}-2\right) q_{A} / 2$ sign-preserving edges. Then $q_{A}=2$ by Lemma 4.6.

Lemma 9.2. Suppose that $\Gamma_{A}$ does not have a non-parallel pair of double boundary edges, and $m_{D}>0$. If $\Delta \geq 4$, then $q_{A}=2$.

Proof. Since $\Gamma_{A}$ does not have a non-parallel pair of double boundary edges, if a vertex of $\widetilde{\Gamma}_{A}$ has two boundary edges incident to it, then they are incident to distinct components of $\partial A$. Thus every vertex of $\widetilde{\Gamma}_{A}$ has at most two boundary edges incident to it. Hence every vertex of $\Gamma_{A}$ has at most $p_{D}+m_{D}=q_{D}$ boundary edges incident to it, by Lemmas 7.1 and 7.2 and the fact that $m_{D}>0$. Thus $\Gamma_{A}$ has at most $q_{D} q_{A}$ boundary edges. The graph $\Gamma_{A}$ contains at most $q_{D} q_{A} / 2$ signpreserving edges by Lemma 4.3. Hence the number of end points of sign-reversing edges is at least

$$
\begin{gathered}
4 q_{D} q_{A}-q_{D} q_{A}-\left(q_{D} q_{A} / 2\right) 2 \\
=\left(2 q_{D}-2\right) q_{A}+2 q_{A} .
\end{gathered}
$$

Thus the parity rule implies that $\Gamma_{D}$ has more than $\left(2 q_{D}-2\right) q_{A} / 2$ sign-preserving edges. Then $q_{A}=2$ by Lemma 4.6 .

\section{Proof of Theorem 1.1 When $\Gamma_{A}$ Contains \\ A NON-PARALLEL PAIR OF DOUBLE INTERIOR EDGES}

In this section we prove Theorem 1.1 when the graph $\Gamma_{A}$ contains a non-parallel pair of double interior edges. That is, the graph $\Gamma_{A}$ contains a pair of non-parallel interior edges $\alpha$ and $\beta$ which connect two distinct vertices $u$ and $v$ and the loop consisting of these edges bounds a disc $E$ on $A$ (see Figure 18).

We take the pair $\alpha$ and $\beta$ so that it is an innermost one among all such pairs of edges on $A$. Then we take the subgraph $G_{A}=\left(\Gamma_{A} \cap E\right)-(\alpha \cup \beta)-$ (loop edges

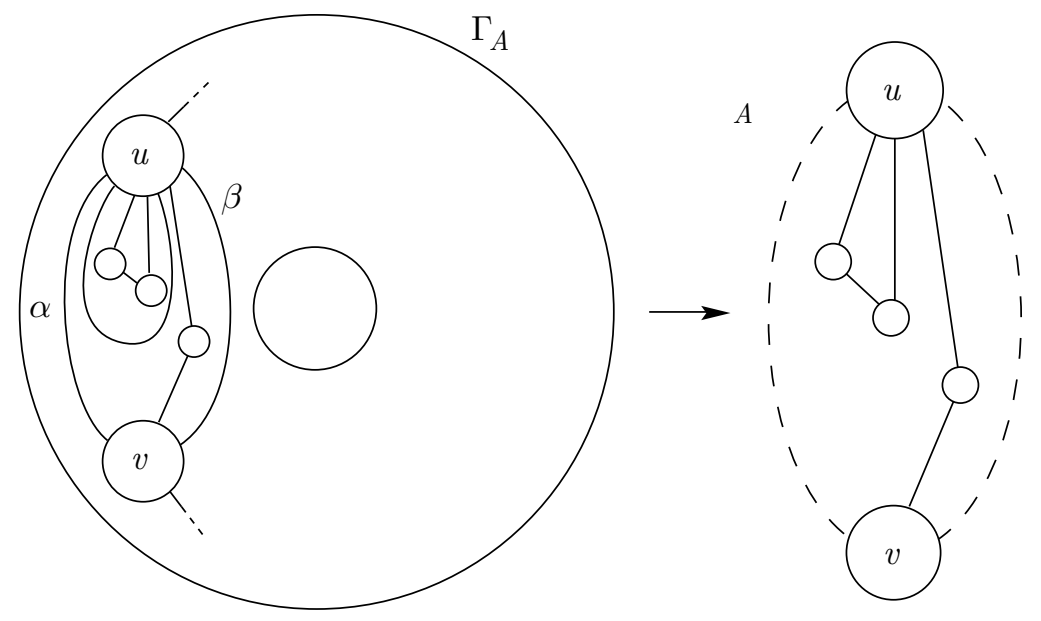

FIGURE 18 
incident to $u$ or $v$ ); see Figure 18. We call the vertices $u$ and $v$ terminal vertices of $G_{A}$, and the other vertices of $G_{A}$ inner vertices. We call the interior edges of $G_{A}$ incident to terminal vertices terminal edges of $G_{A}$, and the interior edges connecting inner vertices of $G_{A}$ inner edges of $G_{A}$.

Proposition 8.1 and Lemmas 7.6, 10.2 and 10.3 form a proof of Theorem 1.1 for this situation.

The next Lemma 10.1 is used also in section 11.

Lemma 10.1. Assume that $q_{D} \geq 2$. Let $x$ be a terminal vertex, and $v_{x}$ be the valency of $x$ in $\widetilde{G}_{A}$. Then either

(1) the knot $K$ is a cable knot in $W$, or

(2) $q_{A}=2$, or

(3) the valency of $x$ in $G_{A}$ is at most $\left(v_{x}+2\right) q_{D} / 2-2$.

Proof. We assume (1) and (2) do not hold and show that (3) holds. The vertex $x$ has at most $2 q_{D}-2$ sign-reversing edges incident to it in the graph $G_{A}$. Otherwise, the graph $\Gamma_{D}$ would have more than $2 q_{D}-2$ sign-preserving $x$-edges by the parity rule, and (2) $q_{A}=2$ by Lemma 4.5 .

Every sign-preserving edge in $\widetilde{G}_{A}$ corresponds to at most $q_{D} / 2$ parallel edges by Lemma 4.7. Every sign-reversing edge in $\widetilde{G}_{A}$ corresponds to at most $q_{D}-1$ parallel edges (otherwise (1) holds by Lemma 4.8).

Let $k_{x}$ be the number of sign-reversing edges incident to $x$ in the reduced graph $\widetilde{G}_{A}$. Then the valency of $x$ in $G_{A}$ is at most

$$
\begin{gathered}
\left(2 q_{D}-2\right)+\left(v_{x}-k_{x}\right) q_{D} / 2=\left(v_{x}-k_{x}+4\right) q_{D} / 2-2 \quad \text { when } k_{x} \geq 2, \\
\left(q_{D}-1\right)+\left(v_{x}-1\right) q_{D} / 2=\left(v_{x}+1\right) q_{D} / 2-1 \quad \text { when } k_{x}=1, \\
v_{x} q_{D} / 2 \quad \text { when } k_{x}=0 .
\end{gathered}
$$

Since $q_{D} \geq 2$, the largest of these values occurs when $k_{x}=2$. Hence we have (3).

Let $r_{A}$ be the number of inner vertices of $G_{A}$. Since $G_{A}$ has two terminal vertices and at least one inner vertex, $\Gamma_{A}$ has at least three vertices. Moreover, because $A$ is separating, $q_{A} \geq 4$.

Lemma 10.2. Assume that $q_{D} \geq 2$. Suppose that the graph $\widetilde{G}_{A}$ contains at most $2 r_{A}-2$ terminal edges. If $\Delta \geq 4$, then the knot $K$ is a cable knot in $W$.

Proof. Since $q_{A} \geq 4, G_{A}$ has at most $\left(2 q_{D}-2\right) r_{A} / 2$ inner sign-reversing edges by the parity rule and similar arguments as in the proof of Lemma 4.6. The graph $G_{A}$ is a graph on the disc $E$ and has at most $\left(r_{A}-1\right) q_{D} / 2$ inner sign-preserving edges by similar arguments as in the proof of Lemmas 4.2 and 4.3 . Then the number of end points of terminal edges at inner vertices is at least

$$
\begin{gathered}
4 q_{D} r_{A}-\left(2 q_{D}-2\right) r_{A}-\left(r_{A}-1\right) q_{D} \\
=q_{D} r_{A}+2 r_{A}+q_{D} .
\end{gathered}
$$

Now we suppose for a contradiction that the $\operatorname{knot} K$ is not a cable knot in $W$. Let $U$ and $V$ be the valencies of the terminal vertices $u$ and $v$ in $\widetilde{G}_{A}$ respectively. Then by Lemma 10.1 and $q_{A} \geq 4$, the number of end points of terminal edges at 


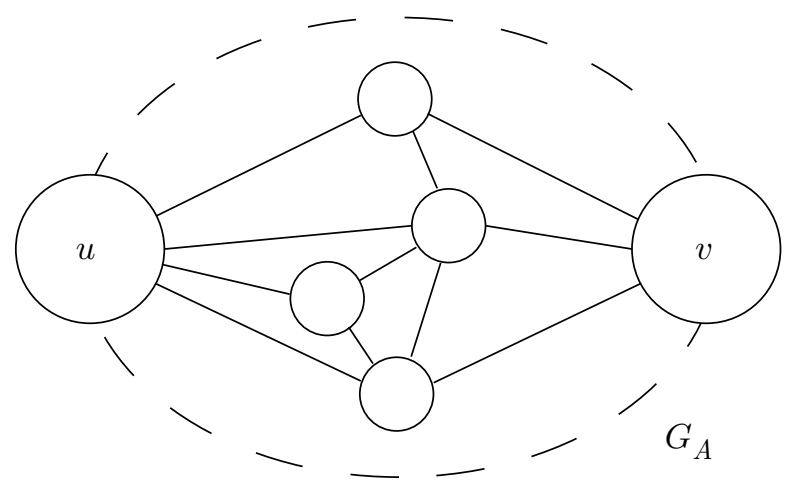

FIGURE 19

inner vertices is at most

$$
\begin{aligned}
\{(U+ & \left.2) q_{D} / 2-2\right\}+\left\{(V+2) q_{D} / 2-2\right\} \\
& =(U+V) q_{D} / 2+2 q_{D}-4 \\
& \leq\left(2 r_{A}-2\right) q_{D} / 2+2 q_{D}-4 \\
& =\left(q_{D} r_{A}+2 r_{A}+q_{D}\right)-\left(2 r_{A}+4\right) .
\end{aligned}
$$

This is a contradiction.

Lemma 10.3. Assume that $q_{D} \geq 2$. Suppose that the graph $\widetilde{G}_{A}$ contains at least $2 r_{A}-1$ terminal edges. If $\Delta \geq 4$, then the knot $K$ is a cable knot in $W$.

Proof. When $r_{A}=1$, the graph $\widetilde{G}_{A}$ has at most two terminal edges and no inner edges. Hence $G_{A}$ has a parallel family of at least $2 q_{D}$ interior edges. Then by Lemma 4.8 the knot $K$ is a cable knot in $W$.

We can assume $r_{A} \geq 2$ in the rest of this proof. We suppose for a contradiction that the knot $K$ is not a cable knot in $W$. Since we have taken $G_{A}$ so that the pair $\alpha$ and $\beta$ is innermost among all non-parallel pairs of double interior edges, each inner vertex of $G_{A}$ has at most two terminal edges. We note that if an inner vertex has exactly two terminal edges, then one of them is incident to $u$ and the other is incident to $v$. Hence all the inner vertices, except possibly for one, have two terminal edges, because $\widetilde{G}_{A}$ contains at least $2 r_{A}-1$ terminal edges. Therefore $\widetilde{G}_{A}$ has at most $r_{A}$ inner edges (see Figure 19). Each of them corresponds to at most $q_{D}-1$ parallel edges by Lemma 4.8 .

Then the number of end points of terminal edges at inner vertices is at least

$$
4 q_{D} r_{A}-2\left(q_{D}-1\right) r_{A}=2 q_{D} r_{A}+2 r_{A} .
$$

On the other hand, let $U$ and $V$ be the valencies of the terminal vertices $u$ and $v$ in $\widetilde{G}_{A}$ respectively. Notice that $U+V \leq 2 r_{A}$, since each inner vertex has at most two terminal edges. Then by Lemma 10.1 and $q_{A} \geq 4$, the number of end points of 
terminal edges at inner vertices is at most

$$
\begin{aligned}
\{(U & \left.+2) q_{D} / 2-2\right\}+\left\{(V+2) q_{D} / 2-2\right\} \\
& =(U+V) q_{D} / 2+2 q_{D}-4 \\
& \leq 2 r_{A} q_{D} / 2+2 q_{D}-4 \\
& =\left(2 q_{D} r_{A}+2 r_{A}\right)-\left\{q_{D}\left(r_{A}-2\right)+2 r_{A}+4\right\} .
\end{aligned}
$$

Since $r_{A} \geq 2$, this is a contradiction.

\section{Proof of Theorem 1.1 When $\Gamma_{A}$ Contains \\ A NON-PARALLEL PAIR OF DOUBLE BOUNDARY EDGES}

In this section we prove Theorem 1.1 when the graph $\Gamma_{A}$ contains a non-parallel pair of double boundary edges, and does not contain a non-parallel pair of double interior edges. The graph $\Gamma_{A}$ contains a non-parallel pair of boundary edges $\gamma$ and $\delta$ which are incident to the same vertex $w$ and to the same component $C$ of $\partial A$. See Figure 20.

Let $E(\subset A)$ be the disc bounded by the loop which consists of the arcs $\gamma$ and $\delta$ and a subarc of $C$. We take $\gamma$ and $\delta$ so that they form an outermost pair of edges on $A$ as above. Then we take the subgraph $H_{A}=\left(\Gamma_{A} \cap D\right)-(\gamma \cup \delta)-($ loop edges incident to $w$ ). We call the vertex $w$ a terminal vertex of $H_{A}$. We define inner vertices, terminal edges and inner edges of $H_{A}$ as in section 10.

Proposition 8.1 and Lemmas 7.6, 11.1 and 11.2 form a proof of Theorem 1.1 for this situation.

Let $s_{A}(>0)$ be the number of inner vertices of $H_{A}$.

Lemma 11.1. Assume that $q_{D} \geq 2$. Suppose that the graph $\Gamma_{A}$ contains a nonparallel pair of double boundary edges, and does not contain a non-parallel pair of double interior edges. If $m_{D}=0$ and $\Delta \geq 4$, then either (1) $q_{A}=2$, or (2) the knots $K$ and $K^{*}$ are parallel to essential simple loops on $T$ in $W$ and $W(K ; \gamma)$ respectively.

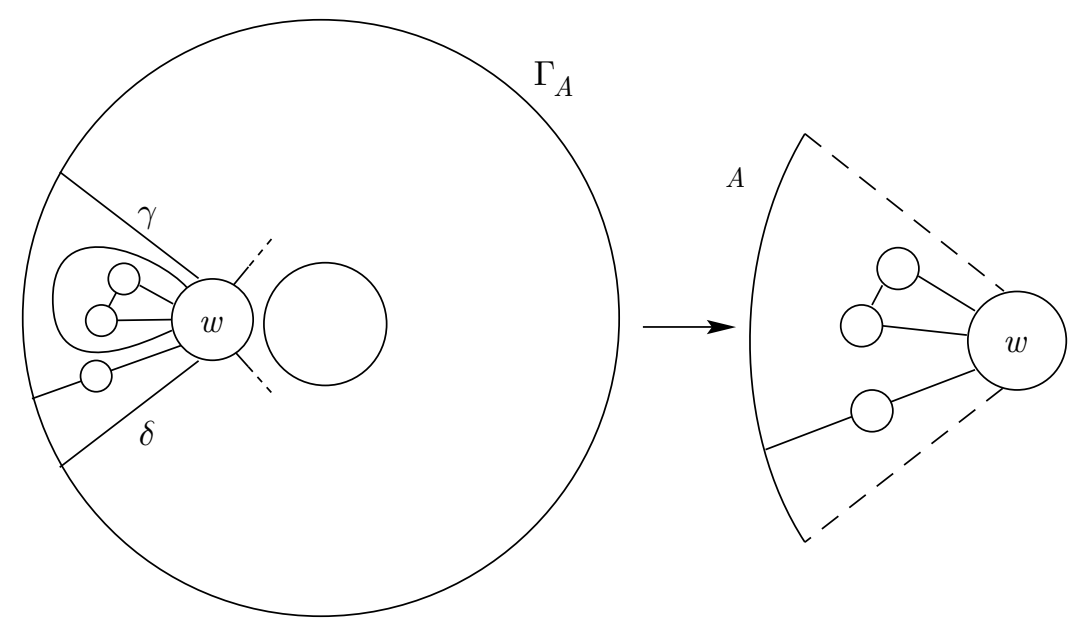

Figure 20 
Proof. We assume that (2) does not hold to show that (1) holds. Since $m_{D}=0$, interior edges of the graph $\Gamma_{D}$ are all sign-preserving edges, and hence the parity rule implies that all the interior edges of $H_{A}$ are sign-reversing. The number of terminal edges is at most $2 q_{D}-2$ by the parity rule and Lemma 4.5 . Every boundary edge of $\widetilde{H}_{A}$ corresponds to at most $2 q_{D}-2$ parallel edges by Lemma 7.5 and $q_{D} \geq 2$. Let $b$ be the number of boundary edges of $\widetilde{H}_{A}$. Let $I$ be the number of end points of inner edges of $H_{A}$. Then we have

$$
I \geq 4 q_{D} s_{A}-(b+1)\left(2 q_{D}-2\right) .
$$

Since we take the pair of edges $\gamma$ and $\delta$ to be outermost, $b \leq s_{A}$. Hence

$$
I \geq\left(2 s_{A}-2\right) q_{D}+2 s_{A}+2>0 .
$$

Thus an inner edge exists, and $b \leq s_{A}-1$. Otherwise, that is, when $b=s_{A}$, Lemma 7.1 and $m_{D}=0$ imply that all the inner vertices of $H_{A}$ would have the same sign, and hence the inner edge is a sign-preserving edge, a contradiction. Hence we have

$$
\begin{aligned}
I \geq & 4 q_{D} s_{A}-\left\{\left(s_{A}-1\right)+1\right\}\left(2 q_{D}-2\right) \\
& =2 q_{D} s_{A}+2 s_{A} \\
& =\left(2 q_{D}-2\right) s_{A}+4 s_{A} .
\end{aligned}
$$

Thus $H_{A}$ has more than $\left(2 q_{D}-2\right) s_{A} / 2$ inner sign-reversing edges. Then the parity rule and similar arguments as in the proof of Lemma 4.6 imply that the condition (1) holds.

Lemma 11.2. Assume that $q_{D} \geq 2$. Suppose that the graph $\Gamma_{A}$ contains a nonparallel pair of double boundary edges, and does not contain a non-parallel pair of double interior edges. If $m_{D}>0$ and $\Delta \geq 4$, then either (1) $q_{A}=2$, or (2) the knot $K$ is a cable knot in $W$.

Proof. When $s_{A}=1$, the graph $\widetilde{H}_{A}$ has at most one terminal edge and at most one boundary edge, and has no inner edges. Hence $H_{A}$ has either a parallel family of at least $2 q_{D}$ interior edges, or a parallel family of at least $2 q_{D}$ boundary edges. In the former case, the knot $K$ is a cable knot in $W$ by Lemma 4.8. In the latter case, we have a contradiction to $m_{D}>0$ by Lemma 7.2.

We can assume $s_{A} \geq 2$ in the rest of this proof. We suppose for a contradiction that neither (1) nor (2) hold. Since $\Gamma_{A}$ does not contain a non-parallel pair of double interior edges, $\widetilde{H}_{A}$ has at most $s_{A}$ terminal edges. Hence $H_{A}$ has at most $\left(s_{A}+2\right) q_{D} / 2-2$ terminal edges by similar arguments as in Lemma 10.1. The graph $H_{A}$ has at most $p_{D} s_{A}$ boundary edges by Lemma 7.2 and the fact that $m_{D}>0$. Every vertex $x$ with the sign - of $\Gamma_{D}$ has at most $s_{A}-1$ sign-reversing edges corresponding to inner edges of $H_{A}$. Otherwise, the graph $H_{A}$ would have more than $s_{A}-1$ inner sign-preserving $x$-edges by the parity rule, and similar arguments as in Lemma 4.2 show that $H_{A}$ would contain a Scharlemann cycle because $H_{A}$ is a graph on the disc $E$. Thus $\Gamma_{D}$ has at most $\left(s_{A}-1\right) m_{D}$ sign-reversing edges corresponding to inner edges of $H_{A}$, and $H_{A}$ has at most $\left(s_{A}-1\right) m_{D}$ inner signpreserving edges by the parity rule. The graph $H_{A}$ has at most $\left(2 q_{D}-2\right) s_{A} / 2$ inner sign-reversing edges by the parity rule and similar arguments as in the proof of Lemma 4.6. Hence the number of end points at inner vertices of $H_{A}$ sums up to 
at most

$$
\begin{aligned}
\left\{\left(s_{A}\right.\right. & \left.+2) q_{D} / 2-2\right\}+p_{D} s_{A}+2 m_{D}\left(s_{A}-1\right)+\left(2 q_{D}-2\right) s_{A} \\
& =4 q_{D} s_{A}-\left(q_{D}-2 m_{D}\right)\left(s_{A} / 2-1\right)-2 s_{A}-2 \\
& <4 q_{D} s_{A} .
\end{aligned}
$$

Note that $p_{D}+m_{D}=q_{D}$ and $s_{A} \geq 2$, and remember that we are assuming that $p_{D} \geq m_{D}$. Thus we have a contradiction.

\section{Dehn surgery CReating an essential annulus with $\Delta=2$}

In this section, we give an example which shows that Theorem 1.1 does not hold for $\Delta=2$. The graphs $\Gamma_{D}$ and $\Gamma_{A}$ defined in section 2 are as shown in Figure 21. The graph $\Gamma_{D}$ has four vertices with the same sign, and the graph $\Gamma_{A}$ has two vertices with distinct signs. Note that $\Gamma_{D}$ contains three Scharlemann cycles for the interval $[1,2]$. A parallel pair of boundary edges of $\Gamma_{A}$ are incident to one component of $\partial A$, and the other parallel pair of boundary edges are incident to the other component of $\partial A$. Let $Q_{1}, Q_{2} \subset P_{A}$ be discs of parallelism of the two pairs of parallel boundary edges of $\Gamma_{A}$. Let $R_{k}$ be the face of $\Gamma_{A}$ which is adjacent to $Q_{k}$ for $k=1$ and 2 .

Let $Y$ be the 3 -manifold obtained by cutting $W$ along the disc $D$. In Figure 22 we see the boundary of the 3 -manifold $Z$ obtained by cutting the exterior $X$ of the knot $K$ along the disc with four holes $P_{D}=D \cap X$. We assume here that $Z$ is irreducible. The regular neighbourhood $V$ of $K$ is cut by the fat vertices of $\Gamma_{D}$ into four 1-handles $H_{[1,2]}, H_{[2,3]}, H_{[3,4]}$ and $H_{[4,1]}$. Let $K_{[k, l]}$ be the arc forming the core of $H_{[k, l]}$. The arc $K_{[1,2]}$ is parallel to the boundary $\partial Y$, where the discs $Q_{1}$ and $Q_{2}$ give discs of parallelism. These discs are parallel in $Z$ because $Z$ is assumed to be irreducible. Let $B_{1} \subset Z$ be the ball between these discs. The arcs $K_{[4,1]}, K_{[3,4]}$ and $K_{[2,3]}$ are parallel in $Y$, where the four discs $Q_{3}, Q_{4}, Q_{5}$ and $Q_{6}$ of parallelism of interior edges of $\Gamma_{A}$ form the discs of parallelism of these arcs. The discs $Q_{3}$ and $Q_{4}$ are parallel in $Z$; let $B_{2}$ be the ball between these discs. The discs $Q_{5}$ and $Q_{6}$ are parallel in $Z$; let $B_{3}$ be the ball between these discs. The closure of $Y-\left(B_{1} \cup H_{[1,2]}\right)$ is homeomorphic to the manifold $Y$. The boundary of the disc $R_{k}(k=1$ or 2$)$ intersects each of the meridian loops of the $\operatorname{arcs} K_{[1,2]}, K_{[4,1]}$ and
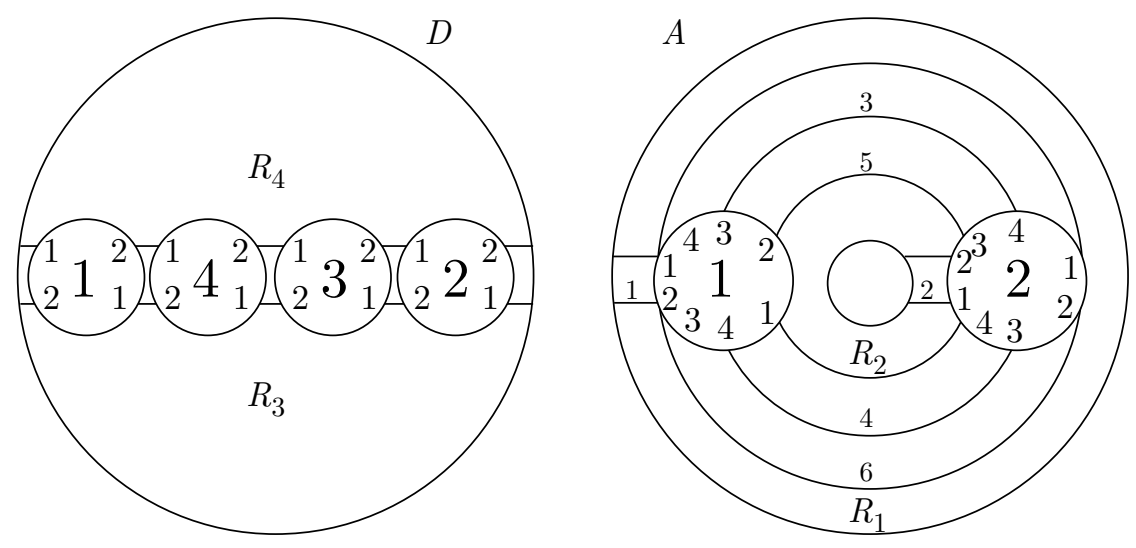

FIGURE 21 


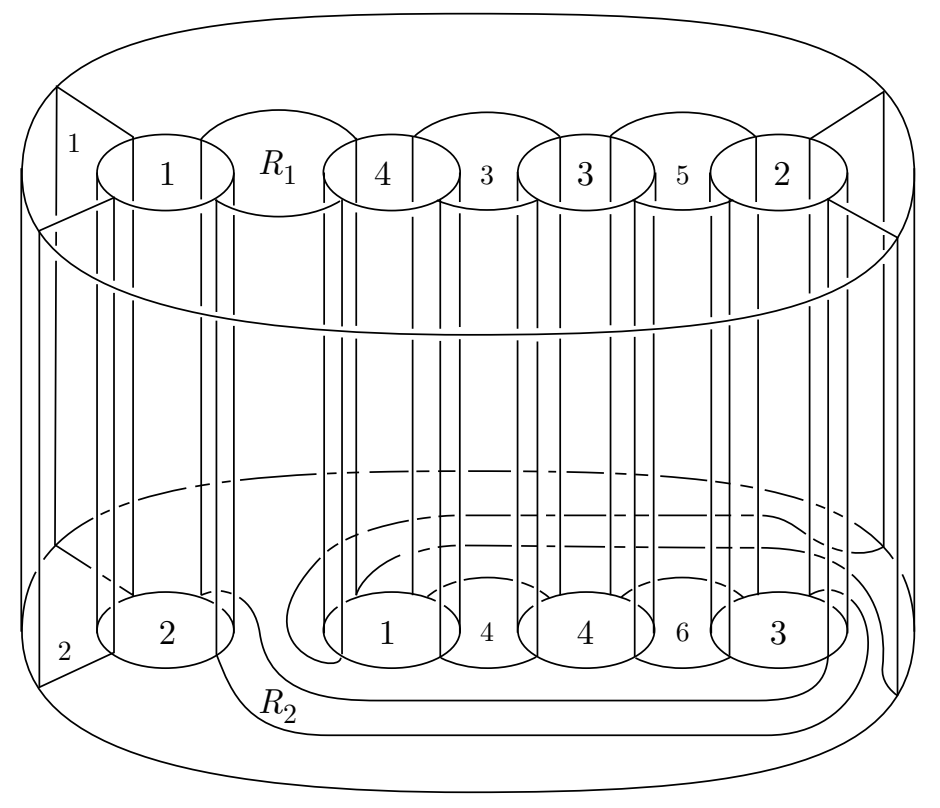

Figure 22

$K_{[2,3]}$ in one point. The closure of $Z-\left(B_{1} \cup B_{2} \cup B_{3}\right)$ has toral boundary, and this boundary is compressed by the discs $R_{1}$ and $R_{2}$. Hence this manifold is a solid torus, and $Y$ is a punctured $\mathbb{R} P^{3}$. The manifold $W$ is the connected sum of a solid torus and a projective space $\mathbb{R} P^{3}$.

Claim 1. $\partial W$ is incompressible in the exterior $X$ of the knot $K$.

Proof. In the manifold $W$ the torus $\partial W$ has a compressing disc $D$. If $W$ contains another compressing disc of $\partial W$, then it is isotopic to $D$ since $W$ is the connected sum of a solid torus and a lens space. Because $D$ intersects $K$ four times algebraically, $X$ does not contain a compressing disc of $\partial W$.

Claim 2. The torus $\partial N(K)$ is incompressible in $X$.

Proof. Suppose for a contradiction that $\partial N(K)$ has a compressing disc $D_{1}$ in $X$. The boundary $\partial D_{1}$ is not a meridian loop of $K$, as otherwise $W$ would contain a nonseparating 2 -sphere. Then the loop $\partial D_{1}$ intersects the disc $D 4 \ell$ times geometrically and algebraically, where $\ell$ is a positive integer. Hence $\partial D_{1}$ represents a non-trivial element of $H_{1}(W, \mathbb{Z})$, which is a contradiction.

Claim 3. The knots $K$ and $K^{*}$ are not parallel to essential simple loops on $\partial W$ and $\partial W(K ; \gamma)$ respectively.

Proof. If either $K$ or $K^{*}$ is parallel to an essential simple loop on $\partial W$ or $\partial W(K ; \gamma)$, then $X$ contains an essential annulus $A_{1}$ whose boundary slope on $\partial N(K)$ is distinct from both meridian slopes of $K$ and $K^{*}$, since $\Delta=2$. Because $D$ intersects $K$ in the minimal number of points up to isotopy, the disc with four holes $P_{D}$ is essential in $X$. Hence we can isotope $A_{1}$ in $X$ so that it intersects $P_{D}$ in essential $\operatorname{arcs}$ on $A_{1}$. Then it follows that the arcs $K_{[4,1]}, K_{[3,4]}$ and $K_{[2,3]}$ are parallel to the boundary in $Y$, which is a contradiction. 
Claim 4. $K$ is not a cable knot in $W$.

Proof. Suppose for a contradiction that $K$ is a cable knot. Let $A_{2}$ be the cabling annulus. We can isotope $A_{2}$ so that it intersects $P_{D}$ in essential arcs on $A_{2}$. Then it follows that the $\operatorname{arc} K_{[1,2]}$ and one of three $\operatorname{arcs} K_{[4,1]}, K_{[3,4]}$ and $K_{[2,3]}$ are parallel in $Y$, which is a contradiction.

Claim 5. The annulus $A$ is incompressible.

Proof. Suppose for a contradiction that $A$ is compressible. Then $\partial W(K ; \gamma)$ is compressible in $W(K ; \gamma)$. We can see that this is a contradiction by Claim 3 and [26, Theorem 2].

The annulus $A$ divides $W(K ; \gamma)$ into two 3 -manifolds $M_{1}$ and $M_{2}$, where $M_{1}$ contains the balls $B_{1}, B_{2}$ and $B_{3}$.

Claim 6. A does not have a $\partial$-compressing disc in $M_{1}$.

Proof. Let $P$ be the closure of a disc face of a Scharlemann cycle of $\Gamma_{D}$. Since $P$ is a Scharlemann disc described in section 3, we have the required result.

Claim 7. A does not have a $\partial$-compressing disc in $M_{2}$.

Proof. If $A$ has a $\partial$-compressing disc in $M_{2}$, then the torus $\partial M_{2}$ is compressible in $M_{2}$. Hence it is sufficient to prove that $\partial M_{2}$ is incompressible in $M_{2}$.

The graph $\Gamma_{D}$ has seven faces: three disc faces of Scharlemann cycles, two discs of parallelism of boundary edges, and the other two discs $R_{3}$ and $R_{4}$. The boundary of the manifold $M_{2}^{\prime}=M_{2} \cap X$ is compressible by the disc $R_{3}$. The closure of $M_{2}-M_{2}^{\prime}$ is a ball, which we regard as a 2-handle. Let $A_{3}=M_{2}^{\prime} \cap \partial N(K)$ : the attaching annulus of this 2-handle. We assume for a contradiction that $\partial M_{2}^{\prime}-A_{3}$ is compressible in $M_{2}^{\prime}$. Let $D_{2}$ be a compressing disc of this surface. We can isotope $D_{2}$ so that it intersects $P_{D}$ in arcs. Let $\alpha$ be an outermost arc on $D_{2}$. This $\operatorname{arc} \alpha$ cobounds an outermost disc together with a subarc $\beta$ of $\partial D_{2}$, and is contained in $R_{3}$ or $R_{4}$. We can assume that $\alpha \subset R_{3}$ without loss of generality. Since $\beta \cap P_{D}=\partial \beta, \alpha$ satisfies the following condition: $\left(^{*}\right) \alpha$ has two end points either in the same interior edge of $\Gamma_{D}$ or in (boundary edges $) \cup\left(\partial D \cap R_{3}\right)$. Among arcs of $D_{2} \cap R_{3}$ satisfying ${ }^{*}$ ) let $\gamma$ be an outermost one on $R_{3}$, and $D_{3}$ the outermost disc of it. We perform surgery on $D_{2}$ along $D_{3}$ and obtain two discs, one of which is a compressing disc of $\partial M_{2}^{\prime}-A_{3}$. Hence by repeating similar operations, we can rechoose the compressing $\operatorname{disc} D_{2}$ so that it does not intersect $P_{D}$. Then $\partial D_{2}$ bounds a disc in $\partial M_{2}^{\prime}-A_{3}$, which is a contradiction. Thus $\partial M_{2}^{\prime}-A_{3}$ is incompressible in $M_{2}^{\prime}$. We can also see that $M_{2}$ has incompressible boundary by the handle addition lemma [18].

Claim 8. $W(K ; \gamma)$ does not contain an essential annulus which is disjoint from $K^{*}$.

Proof. Suppose for a contradiction that $W(K ; \gamma)$ contains such an annulus $A_{4}$. We can isotope $A_{4}$ in the exterior $X$ of the knot $K^{*}$ so that $\left|\partial A_{4} \cap \partial P_{D}\right|$ is minimal and $A_{4} \cap P_{D}$ consists either of essential loops on $A_{4}$ or of essential arcs on $A_{4}$.

In the former case by a standard cut and paste argument we can rechoose the essential annulus $A_{4}$ so that $A_{4} \cap P_{D}=\emptyset$. Then we consider the intersection $A_{4} \cap Q_{1}$, and see that $A_{4}$ is $\partial$-compressible, which is a contradiction.

In the latter case let $D_{4}$ be an arbitrary one of discs of $A_{4} \cap Z$. Then $\partial D_{4} \cap$ $\left(\partial Z \cap P_{D}\right)$ consists of two arcs. This disc $D_{4}$ divides $Y$ into two 3 -manifolds, $M_{3}$ 
and $M_{4}$, one of which, say $M_{3}$, contains the three 1-handles $H_{[4,1]}, H_{[3,4]}$ and $H_{[2,3]}$. Otherwise, $D_{4}$ must intersect at least one of the discs $Q_{3}, Q_{4}, Q_{5}$ and $Q_{6}$, and it follows from a standard cut and paste argument that at least one of the three arcs $K_{[4,1]}, K_{[3,4]}$ and $K_{[2,3]}$ is parallel to the boundary in $Y$, which is a contradiction. Hence every arc of $A_{4} \cap P_{D}$ divides one copy of the disc $D(\subset \partial Y)$ into two discs, of which one contains the fat vertex numbered " 1 " and the other one contains the other vertices numbered " 2 ", " 3 " and " 4 ". This contradicts the fact that every arc of $A_{4} \cap P_{D}$ divides the other copy of the disc $D(\subset \partial Y)$ into two discs of which one contains the fat vertex numbered " 2 " and the other one contains the other vertices numbered "3", "4" and "1".

\section{Proof of Theorem 1.5 AND EXAMPles}

In this final section we prove Theorem 1.5 and present some examples.

Proof of Theorem 1.5. Let us denote the image of $C$ in the resulting manifold $S^{3}(K ; m / n)$ by the same symbol $C$. Take a small fibred tubular neighbourhood $N(C)$ of $C$ in $S^{3}(K ; m / n)$ disjoint from $K^{*}$ (the image of $K$ in $S^{3}(K ; m / n)$ ). Let $W$ be the unknotted solid torus $S^{3}-\operatorname{int} N(C)$. Since $C$ is an exceptional fibre of $S^{3}(K ; m / n), S^{3}(K ; m / n)-\operatorname{int} N(C)=W(K ; m / n)$ is a Seifert fibred manifold over the disc with two exceptional fibres. Let $A$ be a vertical annulus separating the exceptional fibres. Then $A$ is an essential separating annulus in $W(K ; m / n)$. If $\Delta(m / n, 1 / 0)=|n| \geq 4$, then Theorem 1.1 implies that (1) there is a separating essential annulus $A^{\prime}$ such that $A^{\prime} \cap K^{*}=\emptyset$ and $\partial A^{\prime}=\partial A$, or (2) $K$ is parallel to an essential loop on $\partial W$, or (3) $K$ is cabled in $W$. If (1) occurs, then since $\partial A^{\prime}=\partial A$, $A^{\prime}$ is also isotopic to a vertical annulus in $W(K ; m / n)$. (In the following we assume that $A^{\prime}$ is vertical.) We note that $A^{\prime}$ splits $W(K ; m / n)$ into two solid tori, $W_{1}^{\prime}$ and $W_{2}^{\prime}$, and one of them, say $W_{1}^{\prime}$, contains $K^{*}$ in its interior. In addition, since $C$ is an exceptional fibre in $S^{3}(K ; m / n)$, each component of $\partial A^{\prime}(=\partial A)$ is not isotopic to a meridian of $W$. Hence $A^{\prime}$ (regarded as an annulus in $W$ ) also splits $W$ into two solid tori, $W_{1}$ and $W_{2}$, and one of them, say $W_{1}$, contains $K$ in its interior. The core of $W_{1}$ is a cable of the core of $W_{2}$. Then $W_{1}(K ; m / n)=W_{1}^{\prime}$; hence $K$ is a 0 or 1-bridge braid in $W_{1}$ (see [5]). If $K$ is a 0-bridge braid in $W_{1}$ and is not a core of $W_{1}$, then $K$ is cabled in $S^{3}$. If $K$ is a core of $W_{1}$, then $K$ is a cable of the core of $W_{2}$ and hence $K$ is cabled in $S^{3}$. If $K$ is a 1-bridge braid in $W_{1}$, then $|n|=1$ by [5, Proof of Lemma 2.3].

If (2) or (3) occurs, then $K$ is a torus knot or a cable knot. Since the result of Dehn surgery on a trivial knot is a lens space or $S^{3}$, which cannot admit a Seifert fibration over $S^{2}$ with exactly three exceptional fibres, $K$ is non-trivial.

Let us assume that $K$ is a cable of a non-trivial knot. Since $S^{3}(K ; m / n)$ is Seifert fibred over $S^{2}$ with three exceptional fibres and $m / n \neq 0, S^{3}(K ; m / n)$ does not contain an incompressible surface ([17, VI.13.Example]). In particular it is a simple manifold, i.e., $S^{3}(K ; m / n)$ contains no incompressible torus. We take a companion knot $k$ of $K$ that is a simple knot (i.e., a torus knot or a hyperbolic knot). We choose a tubular neighbourhood $V$ of $k$ so that $V$ contains $K$ in its interior. Then from [5] we have three possibilities: (i) $V(K ; m / n) \cong S^{1} \times D^{2}$ and $K$ is a 0 - or 1-bridge braid in $V$, (ii) $V(K ; m / n) \cong\left(S^{1} \times D^{2}\right) \sharp M$, where $M$ is a closed 3-manifold with $1<\left|H_{1}(M ; \mathbb{Z})\right|<\infty$, or (iii) $V(K ; m / n)$ is a $\partial$-irreducible Haken manifold. If (i) or (ii) happens, then $\partial V(K ; m / n)$ is compressible. Therefore $w$ (= the algebraic intersection number of $K$ and a meridian disc of $V$ ) is non-zero 


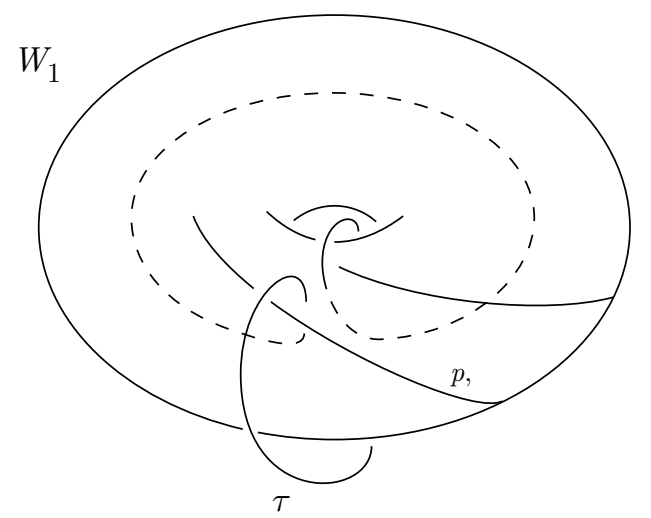

FiguRe 23

([3, Corollary 2.5], [25, 5.2 Corollary]). Now we exclude the possibilities (ii), (iii). Assume for a contradiction that (ii) holds; then $S^{3}(K ; m / n) \cong S^{3}\left(k ; m / n w^{2}\right) \sharp M$ (see [7, Lemma 3.3]). Since $S^{3}(K ; m / n)$ is irreducible, $S^{3}\left(k ; m / n w^{2}\right) \cong S^{3}$. This contradicts Gordon and Luecke's result [12]. If (iii) holds, then $S^{3}(K ; m / n)$ contains an incompressible torus, a contradiction.

Therefore (i) must hold. If $K$ is a 1-bridge braid in $V$, then $|n|=1$ ([5, Proof of Lemma 2.3]). Thus $K$ is a 0 -bridge braid in $V$, and hence $K$ is a cable of a simple knot $k$ with $|w| \geq 2$. Since $S^{3}\left(k ; m / n w^{2}\right) \cong S^{3}(K ; m / n)$ and $\left|n w^{2}\right| \geq 4$, we can apply Theorem 1.1 again for the simple knot $k$ to conclude that $k$ is a torus knot. This completes the proof.

In the rest of this section we describe an infinite family of hyperbolic knots $\left\{K_{p, q, r}\right\}$ each of which satisfies the following property.

By performing a certain Dehn surgery on $K_{p, q, r}$, we obtain a Seifert fibred manifold over $S^{2}$ with three exceptional fibres, and one of them is the image of a knot $C$ which is trivial in $S^{3}$ and is disjoint from $K_{p, q, r}$.

To do that, we first construct knots in solid tori such that certain Dehn surgeries on them produce Seifert fibred manifolds with suitable properties according to [22, Sect.9].

Let $W_{1}$ be a standardly imbedded solid torus in $S^{3}$ with preferred meridianlongitude pair $(M, L)$.

Let $K_{p, q}$ be a simple loop on $\partial W_{1}$ which winds around $p$ times meridionally and $q$ times longitudinally $(q>|p| \geq 2)$. Now we take a trivial knot $\tau$ in $S^{3}$ as depicted in Figure 23. Take a tubular neighbourhood $N(\tau)$ of $\tau$ such that $N(\tau) \cap K_{p, q}=\emptyset$. Let $W=S^{3}-\operatorname{int} N(\tau)$, an unknotted solid torus. Then the loop $K_{p, q}$ is a knot in $W$.

It should be noted that $K_{p, q}$ is a torus knot of type $(p, q)$ in $S^{3}$, and $K_{p, q}$ intersects a meridian disc of $W$ algebraically $(p+q)$-times.

Lemma 13.1. ([22, Lemma 9.1]) The resulting manifold $W\left(K_{p, q} ; p q\right)$ is a Seifert fibred manifold over the disc with two exceptional fibres of indices $|p|, q$. Furthermore, a preferred longitude $L$ of $W$ is a regular fibre of $W\left(K_{p, q} ; p q\right)$.

Twisting along a meridian disc of $W r(\neq 0)$-times, we obtain a new knot $K_{p, q, r} \subset$ $W \subset S^{3}$. Then Lemma 13.1 together with an easy computation yields 
Lemma 13.2. The resulting manifold $W\left(K_{p, q, r} ; p q+(p+q)^{2} r\right)$ is a Seifert fibred manifold over the disc with two exceptional fibres of indices $|p|, q$. Furthermore, $L+r M$ is a regular fibre of $W\left(K_{p, q, r} ; p q+(p+q)^{2} r\right)$.

By extending the Seifert fibration to the complementary solid torus $W^{\prime}=$ $S^{3}$-int $W$ so that the core $C$ is an exceptional fibre of index $|r|$, we see that $S^{3}\left(K_{p, q, r} ; p q+(p+q)^{2} r\right)$ is a Seifert fibred manifold over $S^{2}$ with three exceptional fibres of indices $|p|, q,|r|$. Note that $C$ is a trivial knot in $S^{3}$.

Claim. The complement $S^{3}-K_{p, q, r}$ admits a complete hyperbolic structure of finite volume, provided that $p+q \geq 2$ and $|r|>5$.

Proof. From [22, Claim 9.2], we see that $W-K_{p, q}$ admits a complete hyperbolic structure of finite volume in its interior. The manifold $S^{3}-K_{p, q, r}$ can be obtained from $W-K_{p, q}$ by Dehn filling along the slope $-1 / r$. We recall that if $r=0$, then $K_{p, q, 0}$ is a torus knot and $S^{3}-K_{p, q, 0}$ is not hyperbolic. Since $W-\operatorname{int} N\left(K_{p, q}\right)$ has two boundary components, Gordon's result [8, pp.18-19], [9] asserts that $S^{3}-K_{p, q, r}$ admits a complete hyperbolic structure of finite volume for $|r|>5$.

\section{REFERENCES}

1. Boyer, S. and Zhang, X., The semi-norm and Dehn filling, (preprint).

2. Culler, M., Gordon, C.McA., Luecke, J. and Shalen, P., Dehn surgery on knots, Ann. of Math. 125 (1987), 237-300; 127 (1988), 663. MR 88a:57026; MR 89c:57015

3. Gabai, D., Foliations and the topology of 3-manifolds. II, J. Diff. Geom. 26 (1987), 461-478. MR 89a:57014a

4. $\ldots$, Foliations and the topology of 3-manifolds. III, J. Diff. Geom. 26 (1987), 479-536. MR 89a:57014b

5. _ Surgery on knots in solid tori, Topology 28 (1989), 1-6. MR 90h:57005

6. González-Acuña, F. and Short, H., Knot surgery and primeness, Math. Proc. Camb. Phil. Soc. 99 (1986), 89-102. MR 87c:57003

7. Gordon, C.McA., Dehn surgery and satellite knots, Trans. Amer. Math. Soc. 275 (1983), 687-708. MR 84d:57003

8. __ Dehn surgery on knots, Proceedings ICM Kyoto 1990 (1991), 555-590. MR 93e:57006

9. pear).

10. Gordon, C.McA. and Litherland, R., Incompressible planar surfaces in 3-manifolds, Topology Appl. 18 (1984), 121-144. MR 86e:57013

11. Gordon, C.McA. and Luecke, J., Only integral surgeries can yield reducible manifolds, Math. Proc. Camb. Phil. Soc. 102 (1987), 97-101. MR 89a:57003

12. Knots are determined by their complements, J. Amer. Math. Soc. 2 (1989), 371-415. MR 90a:5700ba

13. _ Reducible manifolds and Dehn surgery, Topology 35 (1996), 385-409. CMP 96:10

14. _ Dehn surgery on knots, Conference in Montreal (1995).

15. Hayashi, C. and Motegi, K., Only single twist on unknots can produce composite knots, Trans. Amer. Math. Soc. 349 (1997), 4465-4479.

16. Hayashi, C. and Shimokawa, K., Symmetric knots satisfy the cabling conjecture, Math. Proc. Camb. Phil. Soc. (to appear).

17. Jaco, W, Lectures on three-manifold topology, CBMS Regional Conf. Ser. Math., no. 43. Amer. Math. Soc., 1980. MR 81k:57009

18. , Adding a 2-handle to a 3-manifold: an application to property R, Proc. Amer. Math. Soc. 92 (1984), 288-292. MR 86b:57006

19. Kouno, M., Motegi, K. and Shibuya, T., Twisting and knot types, J. Math. Soc. Japan 44 (1992), 199-216. MR 93e:57011

20. Luft, E. and Zhang, X., Symmetric knots and the cabling conjecture, Math. Ann. 298 (1994), 489-496. MR 95f:57014

21. Mathieu, Y., Sur des noeuds qui ne sont pas déterminés par leur complément et problémes de cirurgie dans les variétés de dimension 3, Thèse, L'Université de Provence (1990). 
22. Miyazaki, K. and Motegi, K., Seifert fibred manifolds and Dehn surgery, Topology 36 (1997), 579-603. CMP 97:03

23. — Seifert fibred manifolds and Dehn surgery II, (preprint).

24. Morgan, J. and Bass, H. (editors), The Smith conjecture, Pure and Applied Math., vol. 112, Academic Press, 1984. MR 86i:57002

25. Scharlemann, M., Sutured manifolds and generalized Thurston norms, J. Diff. Geom. 29 (1987), 557-614. MR 90c:57021

26. Wu, Y.-Q., Incompressibility of surfaces in surgered 3-manifolds, Topology 31 (1992), 271279. MR 94e:57027

Department of Mathematics, Faculty of Science, Gakushuin University, Mejiro 1-5-1, TOSHIMA-KU, TOKYO 171, JAPAN

E-mail address: chuichiro.hayashi@gakushuin.ac.jp

Department of Mathematics, College of Humanities \& Sciences, Nihon University Sakurajosui 3-25-40, Setagaya-ku, TOKyo 156, Japan

E-mail address: motegi@math.chs.nihon-u.ac.jp 